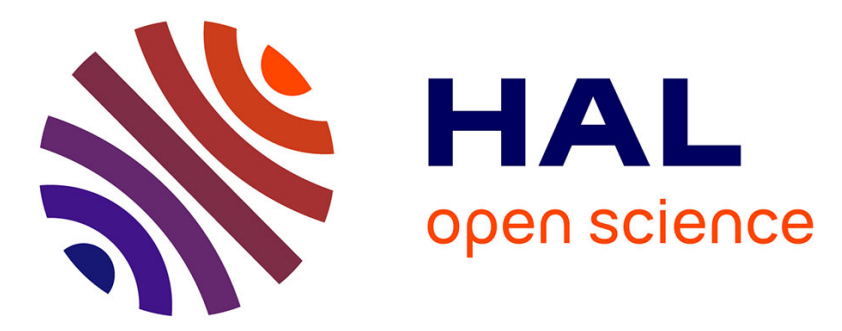

\title{
Low lying eigenvalues and convergence to the equilibrium of some Piecewise Deterministic Markov Processes generators in the small temperature regime
} Arnaud Guillin, Boris Nectoux

\section{- To cite this version:}

Arnaud Guillin, Boris Nectoux. Low lying eigenvalues and convergence to the equilibrium of some Piecewise Deterministic Markov Processes generators in the small temperature regime. 2022. hal02436593v3

\author{
HAL Id: hal-02436593 \\ https://hal.science/hal-02436593v3
}

Preprint submitted on 22 Jan 2022

HAL is a multi-disciplinary open access archive for the deposit and dissemination of scientific research documents, whether they are published or not. The documents may come from teaching and research institutions in France or abroad, or from public or private research centers.
L'archive ouverte pluridisciplinaire HAL, est destinée au dépôt et à la diffusion de documents scientifiques de niveau recherche, publiés ou non, émanant des établissements d'enseignement et de recherche français ou étrangers, des laboratoires publics ou privés. 


\title{
Low lying eigenvalues and convergence to the equilibrium of some Piecewise Deterministic Markov Processes generators in the small temperature regime
}

\author{
Arnaud Guillin* and Boris Nectoux *
}

\begin{abstract}
In this work we study the number of small eigenvalues and the convergence to the equilibrium of the Bouncy Particle Sampler process and the Zig-Zag process generators in the small temperature regime. Such processes, which fall in the class of Piecewise Deterministic Markov Processes, are non diffusive and non reversible. They have recently been used a lot for simulation issues, falling in the domain of Markov Chain Monte Carlo method, due to their numerically observed astonishing performances. Nevertheless, they are far from being theoretically understood, in particular at the spectral level, which is the scope of our study.
\end{abstract}

Keywords. Piecewise Deterministic Markov Processes, metastability, spectral theory, small temperature regime, semiclassical analysis.

AMS classification. 35P15, 35P20, 47F05, 35Q82, 35Q92.

\section{Introduction and main results}

\subsection{Purpose and setting of this work}

\subsubsection{Purpose}

The quite recent growing interest for Piecewise Deterministic Markov Processes 12 (PDMP hereafter) stems from their use within the Markov Chain Monte Carlo methodology. It aims at simulating a target probability distribution $\pi$ by choosing a good Markov chain in the sense that it is ergodic and has stationary probability measure $\pi$. Let us be a little more precise concerning this probability measure $\pi$. Let $\mathrm{M}$ be the position space (for instance, a compact manifold without boundary, which will be the case in this work) and $\pi$ be the Gibbs measure

$$
\pi(d x)=\frac{e^{-\frac{2}{h} U(x)}}{\int_{M} e^{-\frac{2}{h} U}} d x,
$$

associated with the potential function $U: \mathrm{M} \rightarrow \mathbb{R}$ and the parameter $h>0, d x$ being the Lebesgue measure on $\mathrm{M}$. The parameter $h$ is proportional to the Boltzmann constant $k_{B}$ through the relation $h=k_{B} T, T$ being the temperature of the underlying system. The HastingsMetropolis algorithm [32 is surely the most well known method to create such a Markov chain by ensuring reversibility with respect to $\pi$. However, its performance may be questioned in terms of speed of convergence, computational cost, and behavior with respect to the dimension of the problem.

PDMP may be shortly described as follows: between two jumps (possibly of only part of the coordinates) whose rates may of course depend of the position of the process, they have a

*LMBP - Laboratoire de Mathématiques Blaise Pascal, UCA. E-mail: \{arnaud.guillin,boris.nectoux\}@uca.fr 
deterministic behavior (see for instance Remarks 1 and 2 below). PDMP may show remarkable features 4, 18, as they are by essence non reversible and may thus exhibit faster rates of convergence towards equilibrium (see [3]). Of course there is still a lot of work to do to correctly assess the rate of convergence of such PDMP, see for example [1,8,13,16], and the behavior with respect to the dimension still has to be precisely understood (see however [6]). In practice, the second main advantage of these processes is that they can be used to sample the Gibbs measure (1) without sampling Brownian motions, as for example in Langevin type method such as MALA, but only a countable collection of exponentially distributed random variables (using for example thinning procedure). Sampling from such distributions is a major aim of statistical simulations to compute macroscopic quantities or thermodynamic properties, see for instance [31].

However being non reversible, the Hasting-Metropolis trick is no longer useful to guarantee that PDMP have the correct invariant measure. The Bouncy Particle Sampler (BPS) process and the Zig-Zag (ZZ) process fall in the class of Piecewise Deterministic Markov Processes 12 designed to admit $\pi \otimes \nu$ as invariant measure on the space $\mathrm{E}=\mathrm{M} \times \mathrm{V}$, where $\nu$ is an instrumental measure on a space $V$ representing the speed of the process. It is then crucial to study their limiting properties as well as their speed of convergence to equilibrium, which has been the subject of quite a lot of recent and impressive works [2,5, 8, 15, 36]. Let us also mention other contributions: 35] for a spectral analysis in dimension one with a constant jump rate on $\mathbb{T} \times\{ \pm 1\}$, or 7 for a spectral analysis in dimension 1 of the BPS and ZZ processes on the whole line, and [1, 14] for hypocoercive inequalities leading to the convergence of the semigroup to the equilibrium $1_{\mathrm{E}}$ in the weighted $L^{2}$ space $L^{2}(\mathrm{E}, \mathbb{C} ; d \pi d \nu)$. In this work, we provide, to the best of our knowledge, the first study of the spectral properties near the origin of the generators of the BPS process and the ZZ process (see Theorem 11) and its consequences on the rate of convergence to the equilibrium in the small temperature regime $h \rightarrow 0$ (see Theorem 2). The main difficulty arising when studying these generators (see $\mathrm{P}_{h}^{\mathrm{BPS}}$ and $\mathrm{P}_{h}^{\mathrm{ZZ}}$ in the next section) is that they are not symmetric and not diffusive. The main results, namely Theorems 1 and 2 , exhibit a metastable behavior of the processes associated with the operators $\mathrm{P}_{h}^{\mathrm{BPS}}$ and $\mathrm{P}_{h}^{\mathrm{ZZ}}$ : the convergence in $L^{2}(\mathrm{E})$ to the equilibrium $e^{-\frac{1}{h} U} \times \mathbf{1}_{\bigvee}$ is very slow in the small temperature regime.

\subsubsection{Some notations}

In all this work, $M=\mathbb{T}^{d}$ where $\mathbb{T}=\mathbb{R} \backslash \mathbb{Z}$ and $d \geq 1$. Let also $\bigvee$ be either equal to $\{ \pm 1\}^{d}$ or to the $d$-1-dimensional sphere $\mathbb{S}^{d}$ in $\mathbb{R}^{d}$. Denote by $\nu$ the uniform probability measure on $\mathrm{V}$. In all this work, $U: \mathrm{M} \rightarrow \mathbb{R}$ is a $\mathcal{C}^{\infty}$ function on $\mathrm{M}$ and $h>0$ is a parameter which will be referred respectively as the potential function and the temperature of the underlying system. We denote by $\mathrm{E}$ the space $\mathrm{E}=\mathrm{M} \times \mathrm{V}$. We define on $L^{2}(\mathrm{E}, \mathbb{C} ; d x d \nu), d x$ being the Lebesgue measure on $\mathrm{M}$, the operator $\pi_{v}$ by:

$$
\pi_{v} f(x)=\int_{V} f(x, v) d \nu .
$$

Notice that $\pi_{v}$ is a bounded symmetric operator on $L^{2}(\mathrm{E}, d x d \nu)$ and that $\pi_{v}^{2}=\pi_{v}$. Thus $\pi_{v}$ is an orthogonal projection on $L^{2}(\mathrm{E}, \mathbb{C} ; d x d \nu)$. The scalar product on $L^{2}(\mathrm{E}, \mathbb{C} ; d x d \nu)$ is $(f, g) \mapsto \int_{\mathrm{E}} \bar{f} g$. Let $\lambda_{r}: \mathrm{M} \rightarrow \mathbb{R}_{+}$be a non negative bounded function such that:

$$
r_{0}=\inf _{\mathrm{M}} \lambda_{r}>0
$$

In the following, for ease of notation, we denote by $L^{2}(\mathrm{E})$ (resp. $\left.L^{2}(\mathrm{M})\right)$ the space $L^{2}(\mathrm{E}, \mathbb{C} ; d x d \nu)$ (resp. $\left.L^{2}(\mathrm{M}, \mathbb{C} ; d x)\right)$. The Sobolev spaces of higher regularity will be denoted similarly. For instance, $H^{1}(\mathrm{M})$ denotes the set of $u \in L^{2}(\mathrm{M})$ such that $\partial_{x} u \in L^{2}(\mathrm{M})$ (where $\partial_{x} u=$ 
$\left.\left(\partial_{x_{1}} u, \ldots, \partial_{x_{d}} u\right)^{t}\right)$. Finally, we define the set $\mathrm{C}_{x}(\mathrm{E})$ as the set of functions $f \in L^{2}(\mathrm{E})$ such that the distribution $v \cdot \partial_{x} f \in L^{2}(\mathrm{E})$ and for almost every $v \in \mathrm{V}, x \in \mathrm{M} \mapsto f(x, v)$ is $\mathcal{C}^{\infty}$.

\subsubsection{The Bouncy Particle Sampler process generator}

In this section $\mathrm{V}=\mathbb{S}^{d-1}$. For $f \in L^{2}(\mathrm{E})$, the jump operator $\mathrm{B}$ is defined by:

$$
\forall(x, v) \in \mathrm{M} \times \mathrm{V}, \mathrm{B} f(x, v)=f(x, v-2 v \cdot \mathrm{n}(x) \mathrm{n}(x)),
$$

where for all $x \in \mathrm{M}$,

$$
\mathrm{n}(x)=\frac{\partial_{x} U(x)}{\left|\partial_{x} U(x)\right|} \text { if }\left|\partial_{x} U(x)\right| \neq 0, \text { else } \mathrm{n}(x)=0 .
$$

For $h>0, x \in \mathrm{M}$, and $v \in \mathrm{V}$, let

$$
\lambda_{h, \mathrm{~J}}(x, v)=\frac{2}{h}\left(v \cdot \partial_{x} U(x)\right)_{+}
$$

be the jump rate, where the subscript $\mathrm{J}$ stands for jump. Here and in the following, for $a \in \mathbb{R}$, $(a)_{+}$is defined by $(a)_{+}=\max (a, 0)$. Notice that for all $(x, v) \in \mathrm{M} \times \mathrm{V}$, it holds:

$$
\lambda_{h, \mathrm{~J}}(x, v)-\lambda_{h, \mathrm{~J}}(x,-v)=\frac{2}{h} v \cdot \partial_{x} U \quad \text { and } \quad \mathrm{B} \lambda_{h, \mathrm{~J}}(x, v)=\lambda_{h, \mathrm{~J}}(x,-v) .
$$

Let us consider the operator

$$
\mathrm{P}_{h}^{\mathrm{BPS}}=-v \cdot \mathrm{d}_{U, h}+\lambda_{1, \mathrm{~J}}(\mathrm{I}-\mathrm{B})+\lambda_{r}\left(\mathrm{I}-\pi_{v}\right),
$$

where I is the identity operator,

$$
\mathrm{d}_{U, h}=h \partial_{x}+\partial_{x} U=h e^{-\frac{1}{h} U} \partial_{x} e^{\frac{1}{h} U} \text { and } \lambda_{1, J}=2\left(v \cdot \partial_{x} U\right)_{+} .
$$

The formal adjoint of $v \cdot \mathrm{d}_{U, h}$ in $L^{2}(\mathrm{E})$ is the operator $\left(v \cdot \mathrm{d}_{U, h}\right)^{*}=v \cdot\left(-h \partial_{x}+\partial_{x} U\right)=-v \cdot$ $h e^{\frac{1}{h} U} \partial_{x} e^{-\frac{1}{h} U}$. The operator $\mathrm{P}_{h}^{\mathrm{BPS}}$ is linked to the Bouncy Particle Sampler (BPS for short) process generator $\mathrm{L}_{h}^{\mathrm{BPS}}$ where $\mathrm{L}_{h}^{\mathrm{BPS}}=v \cdot \partial_{x}-\lambda_{h, \mathrm{~J}}(\mathrm{I}-\mathrm{B})-\frac{1}{h} \lambda_{r}\left(\mathbf{I}-\pi_{v}\right)$ introduced in 38 . (see also [9, [15, Section 3.1], and Remark 1 below) through the relation

$$
\mathrm{P}_{h}^{\mathrm{BPS}}=-h e^{-\frac{1}{h} U} \mathrm{~L}_{h}^{\mathrm{BPS}} e^{\frac{1}{h} U} .
$$

Using (4), the formal adjoint operator of $\mathrm{P}_{h}^{\mathrm{BPS}}$ in $L^{2}(\mathrm{E})$ is the operator

$$
\left(\mathrm{P}_{h}^{\mathrm{BPS}}\right)^{*}=v \cdot \mathrm{d}_{U, h}+\lambda_{1, \mathrm{~J}}(\cdot,-\cdot)(\mathrm{I}-\mathrm{B})+\lambda_{r}\left(\mathrm{I}-\pi_{v}\right) .
$$

Remark 1. Let us recall the construction of the BPS process $\left(X_{t}^{\mathrm{BPS}}, V_{t}^{\mathrm{BPS}}\right)_{t \geq 0}$ on $\mathbb{T}^{d} \times \mathbb{S}^{d}$. Let $\left(x_{0}, v_{0}\right) \in \mathbb{T}^{d} \times \mathbb{S}^{d}$ and $\left(\mathcal{E}_{1, n}, \mathcal{E}_{2, n}, \mathcal{G}_{n}\right)_{n \in \mathbb{N}^{*}}$ be a family of independent random variables where for $n \geq 1, \mathcal{G}_{n}$ is uniformly distributed on $\mathbb{S}^{d}$ (i.e. with distribution $\nu$ ) and for $q \in\{1,2\}$, $\mathcal{E}_{q, n}$ is an exponential random variable with parameter 1 . Furthermore, for all $n \geq 1, \mathcal{G}_{n}, \mathcal{E}_{1, n}$, and $\mathcal{E}_{2, n}$ are independent. The BPS process $\left(X_{t}^{\mathrm{BPS}}, V_{t}^{\mathrm{BPS}}\right)_{t \geq 0}$ as well as the jump times $\left(\tau_{n}\right)_{n \in \mathbb{N}}$ are then constructed recursively as follows. Set $X_{0}^{\mathrm{BPS}}=x_{0}, V_{0}^{\mathrm{BPS}}=v_{0}$, and $\tau_{0}=0$. Assume that $\left(\tau_{j}\right)_{j=0, \ldots, n}$ and $\left(X_{t}^{\mathrm{BPS}}, V_{t}^{\mathrm{BPS}}\right)_{t \in\left[0, \tau_{n}\right]}$ have been constructed for some $n \geq 0$. Then, consider

$$
\mathrm{t}_{1, n+1}=\inf \left\{t \geq 0, \frac{1}{h} \int_{0}^{t} \lambda_{r}\left(X_{\tau_{n}}^{\mathrm{BPS}}+s V_{\tau_{n}}^{\mathrm{BPS}}\right) d s \geq \mathcal{E}_{1, n+1}\right\}
$$


and

$$
\mathrm{t}_{2, n+1}=\inf \left\{t \geq 0, \int_{0}^{t} \lambda_{h, J}\left(X_{\tau_{n}}^{\mathrm{BPS}}+s V_{\tau_{n}}^{\mathrm{BPS}}, V_{\tau_{n}}^{\mathrm{BPS}}\right) d s \geq \mathcal{E}_{2, n+1}\right\} .
$$

Moreover, set $\mathrm{t}_{n+1}=\min \left(\mathrm{t}_{1, n+1}, \mathrm{t}_{2, n+1}\right)$ and $\tau_{n+1}=\tau_{n}+\mathrm{t}_{n+1}$. Then, for all $t \in\left[\tau_{n}, \tau_{n+1}\right)$,

$$
X_{t}^{\mathrm{BPS}}=X_{\tau_{n}}^{\mathrm{BPS}}+\left(t-\tau_{n}\right) V_{\tau_{n}}^{\mathrm{BPS}} \text { and } V_{t}^{\mathrm{BPS}}=V_{\tau_{n}}^{\mathrm{BPS}} .
$$

Finally, set $X_{\tau_{n+1}}^{\mathrm{BPS}}=X_{\tau_{n}}^{\mathrm{BPS}}+\mathrm{t}_{n+1} V_{\tau_{n}}^{\mathrm{BPS}}$ and

$$
V_{\tau_{n+1}}^{\mathrm{BPS}}= \begin{cases}\mathcal{G}_{n+1} & \text { if } \mathrm{t}_{n+1}=\mathrm{t}_{1, n+1} \\ V_{\tau_{n}}^{\mathrm{BPS}}-2\left(\mathrm{n}\left(X_{\tau_{n+1}}^{\mathrm{BPS}}\right) \cdot V_{\tau_{n}}^{\mathrm{BPS}}\right) \mathrm{n}\left(X_{\tau_{n+1}}^{\mathrm{BPS}}\right) & \text { otherwise, }\end{cases}
$$

where $\mathrm{n}$ is defined in (3).

\subsubsection{The Zig Zag process generator}

Let $\left(\mathrm{e}_{1}, \ldots, \mathrm{e}_{d}\right)$ be the canonical basis of $\mathbb{R}^{d}$. When $\mathrm{V}=\mathbb{S}^{d-1}$, for $f \in L^{2}(\mathrm{E})$, one defines for $k \in\{1, \ldots, d\}$, the jump operator $\mathrm{B}^{(k)}$ as:

$$
\forall(x, v) \in \mathrm{M} \times \mathrm{V}, \mathrm{B}^{(k)} f(x, v)=f\left(x, v-2 v \cdot \mathrm{n}^{(k)}(x) \mathrm{n}^{(k)}(x)\right),
$$

where $\mathbf{n}^{(k)}$ is defined by: for all $x \in \mathbf{M}$,

$$
\mathbf{n}^{(k)}(x)=\frac{\partial_{x_{k}} U(x)}{\left|\partial_{x_{k}} U(x)\right|} \mathrm{e}_{k} \quad \text { if }\left|\partial_{x_{k}} U(x)\right| \neq 0, \quad \text { else } \mathbf{n}^{(k)}(x)=0 .
$$

When $\mathrm{V}=\{ \pm 1\}^{d}$, for all $k \in\{1, \ldots, d\}$, the jump operator $\mathrm{B}^{(k)}$ is defined for $f \in L^{2}(\mathrm{E})$, by:

$$
\forall(x, v) \in \mathrm{M} \times \mathrm{V}, \mathrm{B}^{(k)} f(x, v)=f\left(x, v-2 v \cdot \mathrm{e}_{k} \mathrm{e}_{k}\right) .
$$

In this case, $\mathrm{B}^{(k)}$ consists in negating the $k$-th component of $v \in\{ \pm 1\}^{d}$. For $h>0,(x, v) \in \mathrm{M} \times \mathrm{V}$, and $k \in\{1, \ldots, d\}$, let

$$
\lambda_{h, \mathrm{~J}}^{(k)}(x, v)=\frac{2}{h}\left(v \cdot \partial_{x_{k}} U(x) \mathrm{e}_{k}\right)_{+}
$$

be the $k$-th jump rate. Notice that for all $\forall(x, v) \in \mathrm{M} \times \mathrm{V}$, it holds for the two previous cases:

$$
\lambda_{h, \mathrm{~J}}^{(k)}(x, v)-\lambda_{h, \mathrm{~J}}^{(k)}(x,-v)=\frac{2}{h} v \cdot \partial_{x_{k}} U(x) \mathrm{e}_{k} \quad \text { and } \quad \mathrm{B}^{(k)} \lambda_{h, \mathrm{~J}}^{(k)}(x, v)=\lambda_{h, \mathrm{~J}}^{(k)}(x,-v) .
$$

Let us consider the operator

$$
\mathrm{P}_{h}^{\mathrm{ZZ}}=-v \cdot \mathrm{d}_{U, h}+\sum_{k=1}^{d} \lambda_{1, J}^{(k)}\left(\mathrm{I}-\mathrm{B}^{(k)}\right)+\lambda_{r}\left(\mathrm{I}-\pi_{v}\right),
$$

where $\lambda_{1, J}^{(k)}(x, v)=2\left(v \cdot \partial_{x_{k}} U(x) \mathrm{e}_{k}\right)_{+}$. The operator $\mathrm{P}_{h}^{Z Z}$ is linked to the Zig-Zag (ZZ for short) process generator $\mathrm{L}_{h}^{\mathrm{ZZ}}$ where $\mathrm{L}_{h}^{\mathrm{ZZ}}=v \cdot \partial_{x}-\sum_{k=1}^{d} \lambda_{h, \mathrm{~J}}^{(k)}\left(\mathrm{I}-\mathrm{B}^{(k)}\right)-\frac{1}{h} \lambda_{r}\left(\mathbf{I}-\pi_{v}\right)$ through the relation

$$
\mathrm{P}_{h}^{\mathrm{ZZ}}=-h e^{-\frac{1}{h} U} \mathbf{L}_{h}^{\mathrm{ZZ}} e^{\frac{1}{h} U} .
$$

We refer to [5] and references therein for more details on the ZZ process (see also [17] and Remark 2 below). Using (5), the formal adjoint operator of $\mathrm{P}_{h}^{Z Z}$ in $L^{2}(\mathrm{E})$ is the differential operator

$$
\left(\mathrm{P}_{h}^{\mathrm{ZZ}}\right)^{*}=v \cdot \mathrm{d}_{U, h}+\sum_{k=1}^{d} \lambda_{1, \mathrm{~J}}^{(k)}(\cdot,-\cdot)(\mathrm{I}-\mathrm{B})+\lambda_{r}\left(\mathrm{I}-\pi_{v}\right) .
$$


Remark 2. Let us recall the construction of the $\mathrm{ZZ}$ process. Let us for instance assume that $\mathrm{V}=\{ \pm 1\}^{d}$. Let $\left(x_{0}, v_{0}\right) \in \mathbb{T}^{d} \times\{ \pm 1\}^{d}$ and $\left(\mathcal{E}_{1, n}, \mathcal{E}_{2, n}^{(k)}, \mathcal{G}_{n}\right)_{n \in \mathbb{N}^{*}, k=1, \ldots, d}$ be a family of independent random variables where for $n \geq 1, \mathcal{G}_{n}$ is uniformly distributed on $\{ \pm 1\}^{d}$, and $\mathcal{E}_{1, n}$ and $\mathcal{E}_{2, n}^{(k)}$ $(k \in\{1, \ldots, d\})$ are exponential random variables with parameter 1 . Furthermore, for all $n \geq 1$ and all $k \neq \ell, \mathcal{G}_{n}, \mathcal{E}_{1, n}, \mathcal{E}_{2, n}^{(k)}$, and $\mathcal{E}_{2, n}^{(\ell)}$ are independent. The $\mathrm{ZZ}$ process $\left(X_{t}^{\mathrm{ZZ}}, V_{t}^{\mathrm{ZZ}}\right)_{t \geq 0}$ as well as the jump times $\left(\tau_{n}\right)_{n \in \mathbb{N}}$ are then constructed recursively as follows. Set $X_{0}^{\mathrm{ZZ}}=x_{0}, V_{0}^{\mathrm{ZZ}}=v_{0}$, and $\tau_{0}=0$. Assume that $\left(\tau_{j}\right)_{j=0, \ldots, n}$ and $\left(X_{t}^{\mathrm{ZZ}}, V_{t}^{\mathrm{ZZ}}\right)_{t \in\left[0, \tau_{n}\right]}$ have been constructed for some $n \geq 0$. Then, consider

$$
\mathrm{t}_{1, n+1}=\inf \left\{t \geq 0, \frac{1}{h} \int_{0}^{t} \lambda_{r}\left(X_{\tau_{n}}^{\mathrm{ZZ}}+s V_{\tau_{n}}^{\mathrm{ZZ}}\right) d s \geq \mathcal{E}_{1, n+1}\right\}
$$

and for $k \in\{1, \ldots, d\}$,

$$
\mathrm{t}_{2, n+1}^{(k)}=\inf \left\{t \geq 0, \int_{0}^{t} \lambda_{h, \mathrm{~J}}^{(k)}\left(X_{\tau_{n}}^{\mathrm{ZZ}}+s V_{\tau_{n}}^{\mathrm{ZZ}}, V_{\tau_{n}}^{\mathrm{ZZ}}\right) d s \geq \mathcal{E}_{2, n+1}^{(k)}\right\}, \quad k_{0}=\underset{k \in\{1, \ldots, d\}}{\arg \min } \mathrm{t}_{2, n+1}^{(k)} .
$$

In addition, set $\mathrm{t}_{n+1}=\min \left(\mathrm{t}_{1, n+1}, \mathrm{t}_{2, n+1}^{\left(k_{0}\right)}\right)$ and $\tau_{n+1}=\tau_{n}+\mathrm{t}_{n+1}$. Then, for all $t \in\left[\tau_{n}, \tau_{n+1}\right)$,

$$
X_{t}^{\mathrm{ZZ}}=X_{\tau_{n}}^{\mathrm{ZZ}}+\left(t-\tau_{n}\right) V_{\tau_{n}}^{\mathrm{ZZ}} \text { and } V_{t}^{\mathrm{ZZ}}=V_{\tau_{n}}^{\mathrm{ZZ}} .
$$

Finally, set $X_{\tau_{n+1}}^{\mathrm{ZZ}}=X_{\tau_{n}}^{\mathrm{ZZ}}+\mathrm{t}_{n+1} V_{\tau_{n}}^{\mathrm{ZZ}}$ and

$$
V_{\tau_{n+1}}^{\mathrm{ZZ}}= \begin{cases}\mathcal{G}_{n+1} & \text { if } \mathrm{t}_{n+1}=\mathrm{t}_{1, n+1} \\ V_{\tau_{n}}^{\mathrm{ZZ}}-2\left(\mathrm{e}_{k_{0}} \cdot V_{\tau_{n}}^{\mathrm{ZZ}}\right) \mathrm{e}_{k_{0}} & \text { otherwise. }\end{cases}
$$

Remark 3. Notice that $\left(\mathrm{P}_{h}^{\mathrm{BPS}}\right)^{*}\left(e^{-\frac{1}{h} U} \mathbf{1}_{\mathrm{V}}\right)=\left(\mathrm{P}_{h}^{\mathrm{ZZ}}\right)^{*}\left(e^{-\frac{1}{h} U} \mathbf{1}_{\mathrm{V}}\right)=0$ and thus, the measure $\pi \otimes \nu$ is invariant for both the BPS process and the $\mathrm{ZZ}$ process, where $\pi$ is the Gibbs measure (1).

Remark 4. Let us explain the choice of scaling in $h$ in the refreshment operator $\mathrm{R}_{v}=\frac{1}{h} \lambda_{r}\left(\mathrm{I}-\pi_{v}\right)$. This scaling is explained by the fact that in practice, a refreshment is added to balance the jump rate $\lambda_{h, \mathrm{~J}}$ (or the $\lambda_{h, \mathrm{~J}}^{(k)}$ 's when considering the ZZ process) in order to sample efficiently the measure $\pi \otimes \nu$. On the other hand, if the refreshment is too large compared to $\lambda_{h, \mathrm{~J}}$, the convergence rate towards $\pi \otimes \nu$ becomes very poor. There is a trade-off between the added refreshment and $\lambda_{h, \mathrm{~J}}$ (see for example [13, 16] for some very partial explanation). The relevant scaling when $h \ll 1$ of $\mathrm{R}_{v}$ is thus of the same order of $\lambda_{h, \mathrm{~J}}$ which scales in $h^{-1}$. Other scalings for the refreshment operator are considered in Section 5.3 below.

\subsection{Assumptions and main results}

The following result will be needed.

Lemma 5. For all $w \in \mathrm{C}_{x}(\mathrm{E})$, it holds:

$$
\operatorname{Re}\left\langle\mathrm{P}_{h}^{\mathrm{BPS}} u, u\right\rangle=\frac{1}{2} \int_{\mathrm{E}} \lambda_{1, \mathrm{~J}}|\mathrm{~B} u-u|^{2}+\int_{\mathrm{M}} \lambda_{r} \int_{\mathrm{V}}\left|\left(\mathrm{I}-\pi_{v}\right) u\right|^{2},
$$

and

$$
\operatorname{Re}\left\langle\mathrm{P}_{h}^{Z Z} u, u\right\rangle=\frac{1}{2} \sum_{k=1}^{d} \int_{\mathrm{E}} \lambda_{1, \mathrm{~J}}^{(k)}\left|\mathrm{B}^{(k)} u-u\right|^{2}+\int_{\mathrm{M}} \lambda_{r} \int_{\mathrm{V}}\left|\left(\mathrm{I}-\pi_{v}\right) u\right|^{2}
$$


Proof. Let us prove (6). The other equality is proved similarly. Let us consider $w \in \mathrm{C}_{x}(\mathrm{E})$. Then, one has:

$$
\left\langle\mathrm{P}_{h} w, w\right\rangle=\int_{\mathrm{E}}\left(-v \cdot \mathrm{d}_{U, h} \bar{w}\right) w+\int_{\mathrm{E}} \lambda_{1, \mathrm{~J}}(\mathrm{I}-\mathrm{B}) \bar{w} w+\int_{\mathrm{M}} \lambda_{r} \underbrace{\int_{\mathrm{V}}\left(\mathrm{I}-\pi_{v}\right) \bar{w} w d \nu}_{=\int_{\mathrm{V}}\left|\left(\mathrm{I}-\pi_{v}\right) w\right|^{2} d \nu} d \pi .
$$

Since for almost every $v \in \mathrm{V}, x \in \mathrm{M} \mapsto w(x, v)$ is smooth, it holds: $\mathrm{d}_{U, h} \bar{w}=h \partial_{x} \bar{w}+\partial_{x} U \bar{w}$ and

$$
\int_{\mathrm{E}} v \cdot \partial_{x}\left(|w|^{2}\right)=\int_{\mathrm{E}} v \cdot\left[\left(\partial_{x} \bar{w}\right) w+\left(\partial_{x} w\right) \bar{w}\right] .
$$

By the Stokes Theorem $\int_{\mathbf{M}} v \cdot \partial_{x}\left(|w|^{2}\right) d x=0$, and therefore, one has:

$$
\int_{\mathrm{E}} v \cdot \operatorname{Re}\left(\left(\partial_{x} \bar{w}\right) w\right)=0
$$

and then, $\operatorname{Re} \int_{\mathrm{E}}\left(-v \cdot \mathrm{d}_{U, h} \bar{w}\right) w=\int_{\mathrm{E}}-v \cdot \partial_{x} U|w|^{2}$. Let us recall that $\mathrm{B}$ is symmetric on $L^{2}(\mathrm{E})$. Besides, one has:

$$
\begin{aligned}
\operatorname{Re} \int_{\mathrm{E}} \lambda_{1, \mathrm{~J}}(\mathrm{I}-\mathrm{B}) \bar{w} w= & \frac{1}{2} \int_{\mathrm{E}}\left[\lambda_{1, \mathrm{~J}}(\bar{w}-\mathrm{B} \bar{w}) w+\lambda_{1, \mathrm{~J}}(w-\mathrm{B} w) \bar{w}\right] \\
= & \frac{1}{2} \int_{\mathrm{E}}\left[\lambda_{1, \mathrm{~J}}|w-\mathrm{B} w|^{2}+\lambda_{1, \mathrm{~J}}(\bar{w}-\mathrm{B} \bar{w}) \mathrm{B} w+\lambda_{1, \mathrm{~J}}(\cdot,-\cdot)(\mathrm{B} w-w) \mathrm{B} \bar{w}\right] \\
= & \frac{1}{2} \int_{\mathrm{E}} \lambda_{1, \mathrm{~J}}|w-\mathrm{B} w|^{2}+\frac{1}{2} \int_{\mathrm{E}}\left(\lambda_{1, \mathrm{~J}}(\cdot,-\cdot)-\lambda_{1}\right)|\mathrm{B} w|^{2} \\
& +\int_{\mathrm{E}}\left(\lambda_{1, \mathrm{~J}} \overline{\mathrm{w}} \mathrm{B} w-\lambda_{1, \mathrm{~J}}(\cdot,-\cdot) w \mathrm{~B} \bar{w}\right) .
\end{aligned}
$$

Since $\int_{\mathrm{E}}\left(\lambda_{1, J} \overline{\mathrm{w}} \mathrm{B} f-\lambda_{1, \mathrm{~J}}(\cdot,-\cdot) w \mathrm{~B} \bar{w}\right)=0$, one has:

$$
\operatorname{Re} \int_{\mathrm{E}} \lambda_{1, \mathrm{~J}}(\mathrm{I}-\mathrm{B}) \bar{w} w=\frac{1}{2} \int_{\mathrm{E}} \lambda_{1, \mathrm{~J}}|w-\mathrm{B} w|^{2}+\frac{1}{2} \int_{\mathrm{E}}\left(\lambda_{1, \mathrm{~J}}-\lambda_{1, \mathrm{~J}}(\cdot,-\cdot)\right)|w|^{2} .
$$

Using (4) and the definition of $\lambda_{1, J}$, one obtains:

$$
\frac{1}{2} \int_{\mathrm{E}}\left(\lambda_{1, \mathrm{~J}}-\lambda_{1, \mathrm{~J}}(\cdot,-\cdot)\right)|w|^{2}=\int_{\mathrm{E}} v \cdot \partial_{x} U|w|^{2}=-\operatorname{Re} \int_{\mathrm{E}}\left(-v \cdot \mathrm{d}_{U, h} \bar{w}\right) w .
$$

This concludes the proof of Lemma 5 .

In all this work, we denote by $\mathrm{P}_{h}\left(\right.$ resp. $\left.\mathrm{L}_{h}\right)$ for either $\mathrm{P}_{h}^{\mathrm{BPS}}$ and $\mathrm{P}_{h}^{Z Z}$,

$$
\mathrm{P}_{h} \in\left\{\mathrm{P}_{h}^{\mathrm{BPS}}, \mathrm{P}_{h}^{\mathrm{ZZ}}\right\} \quad\left(\text { resp. } \mathrm{L}_{h} \in\left\{\mathrm{L}_{h}^{\mathrm{BPS}}, \mathrm{L}_{h}^{\mathrm{ZZ}}\right\}\right) .
$$

Let us define

$$
D\left(\mathrm{P}_{h}\right)=\left\{f \in L^{2}(\mathrm{E}), v \cdot \partial_{x} f \in L^{2}(\mathrm{E})\right\} .
$$

The choice of the domain $D\left(\mathrm{P}_{h}\right)$ follows from the fact that $\mathrm{P}_{h}$ is the sum of the unbounded operator $-v \cdot \partial_{x} f$ and a bounded operator on $L^{2}(\mathrm{E})$ (because the jump rates are bounded on $\mathrm{E}$ as well as $\lambda_{r}$ on $\left.\mathbf{M}\right)$. The following lemma is the starting point of our analysis.

Proposition 6. Let $h>0$ be fixed. The space $\mathrm{C}_{x}(\mathrm{E})$ is dense in $D\left(\mathrm{P}_{h}\right)$. Moreover, the operator $\left(\mathrm{P}_{h}, D\left(\mathrm{P}_{h}\right)\right)$ is m-accretive and its adjoint is the operator $\left(\mathrm{P}_{h}^{*}, D\left(\mathrm{P}_{h}^{*}\right)\right)$ with $D\left(\mathrm{P}_{h}^{*}\right)=D\left(\mathrm{P}_{h}\right)$. In addition, $\mathrm{C}^{\infty}(\mathrm{E})$ is a core for both $\mathrm{P}_{h}$ and $\mathrm{P}_{h}^{*}$. 
The proof of this result is postponed to Section 2

Proposition 6 is required for two reasons. The first one is that we need some regularity on test functions to perform computations for $\mathrm{P}_{h}$ (see Lemma 5 for instance and the proof of Proposition 12) and then pass to the limit, to extend these estimates on $D\left(\mathrm{P}_{h}\right)$ in order to obtain resolvent estimates. The second one is that we need to be able to identify the adjoint of $\left(\mathrm{P}_{h}, D\left(\mathrm{P}_{h}\right)\right)$ and to do the same computations for the adjoint as those we did for $\mathrm{P}_{h}$. This is indeed needed to justify that the resolvent of the operator defined by $(30)$ below exist: ${ }^{1}{ }^{1}$ in Step 1 in the proof of Proposition 13 .

Let us now state the main assumption of this work:

Assumption (Morse). The function $U: \mathrm{M} \rightarrow \mathbb{R}$ is a $\mathcal{C}^{\infty}$ Morse function with $\mathrm{m}_{0}$ local minima in $\mathrm{M}$.

The first main result of this work is the following.

Theorem 1. Let us assume that (Morse) holds. Then, there exists $\alpha_{0}>0$ such that for all $\alpha \in\left(0, \alpha_{0}\right)$, there exists $h_{0}>0$ such that for all $h \in\left(0, h_{0}\right)$, the spectrum of $\mathrm{P}_{h}$ is composed of exactly $\mathrm{m}_{0}$ eigenvalues $\left\{\lambda_{1}, \ldots, \lambda_{\mathrm{m}_{0}}\right\}$ (counted with algebraic multiplicity), with $\lambda_{1}=0$, in the set $\left\{\operatorname{Re} \mathbf{z} \leq \alpha h^{2}\right\}$. Moreover, for all $h$ small enough and for all $i \in\left\{1, \ldots, \mathrm{m}_{0}\right\}, \lambda_{i} \in \mathbb{R}$, the algebraic multiplicity of $\lambda_{i}$ equals its geometric multiplicity, and there exists $c>0$ such that for $h$ small enough, $\lambda_{i} \leq e^{-\frac{c}{h}}$. Finally, the eigenvalue 0 has algebraic multiplicity 1 for $\mathrm{P}_{h}$ and Ker $\mathrm{P}_{h}=\operatorname{Span}\left(e^{-\frac{1}{h} U} \mathbf{1}_{\mathrm{V}}\right)$. The same holds for $\mathrm{P}_{h}^{*}$.

The fact that the eigenvalue 0 is simple and isolated for both $\mathrm{P}_{h}$ and $\mathrm{P}_{h}^{*}$ actually holds for all $h>0$, see indeed Proposition 16 in Appendix A. The proof mixes different techniques of semiclassical analysis from [25,30,39]. Our analysis is also inspired from non semiclassical hypocoercive techniques from the original papers 24,26 which were later generalized in 14 (see also [1]).

From Proposition 6, the Hille-Yosida Theorem implies that $-\mathrm{P}_{h}$ generates a strongly continuous contraction semigroup $\left(e^{-t \mathrm{P}_{h}}\right)_{t \geq 0}$ on $L^{2}(\mathrm{E})$. The second main results of this work is the following which characterizes the convergence of the semigroup $\left(e^{-t \mathrm{P}_{h}}\right)_{t \geq 0}$ in the small temperature regime.

Theorem 2. Assume that (Morse) holds. Denote by $\lambda_{1}, \ldots, \lambda_{\mathrm{m}_{0}}$ the $\mathrm{m}_{0}$ smallest eigenvalues of $\mathrm{P}_{h}$, which are real and exponentially small when $h \rightarrow 0$ (see Theorem 1). Let $\Pi_{\lambda_{j}}\left(\mathrm{P}_{h}\right)$, $j=1, \ldots, \mathrm{m}_{0}$, be the spectral projection associated with $\lambda_{j}$ for $\mathbf{P}_{h}$. Then there exist $\gamma>0$, $C>0$, and $h_{0}>0$ such that for all $h \in\left(0, h_{0}\right)$, it holds for all $t \geq 0$ :

$$
\left\|e^{-t \mathrm{P}_{h}}-\sum_{j=1}^{\mathrm{m}_{0}} e^{-t \lambda_{j}} \Pi_{\lambda_{j}}\left(\mathrm{P}_{h}\right)\right\| \leq C e^{-\gamma t h^{2}}
$$

and for all $j=1, \ldots, \mathrm{m}_{0},\left\|\Pi_{\lambda_{j}}\left(\mathrm{P}_{h}\right)\right\| \leq C$.

Here and in the following $\|\mathrm{K}\|$ denotes the norm of $\mathrm{K} \in \mathcal{L}\left(\mathrm{L}^{2}(\mathrm{E})\right)$ when $L^{2}(\mathrm{E})$ is endowed with its natural Hermitian inner product.

In Appendix B below, we give a more precise upper bound on $\lambda_{2}$ (the spectral gap) when $h$ is small enough. More precisely, we show that $\lambda_{2} \leq C h^{-2} \mu_{2}$ where $\mu_{2}$ is the second smallest eigenvalue of the Witten Laplacian $\Delta_{U, h}$ on $\mathrm{M}$ (this operator is introduced in Section 3 below).

\footnotetext{
${ }^{1}$ Using the identity $\operatorname{Ran}(\mathrm{K}-z)=\operatorname{Ker}\left(\mathrm{K}^{*}-\bar{z}\right)^{\perp}$ valid for an accretive, closed, and densely defined operator $\mathrm{K}$ on a Hilbert space.
} 


\section{Proof of Proposition 6}

Let us first prove the following result.

Lemma 7. Let $f \in L^{2}\left(\mathbb{R}_{x}^{d} \times \mathrm{V}\right)$ such that $v \cdot \partial_{x} f \in L^{2}\left(\mathbb{R}_{x}^{d} \times \mathrm{V}\right)$. Then, there exists a sequence $\left(f_{n}\right)_{n \geq 0} \in \mathrm{C}_{x}\left(\mathbb{R}_{x}^{d} \times \mathrm{V}\right)^{\mathbb{N}}$ such that $f_{n} \rightarrow f$ and $v \cdot \partial_{x} f_{n} \rightarrow v \cdot \partial_{x} f$ as $n \rightarrow+\infty$ in $L^{2}\left(\mathbb{R}_{x}^{d} \times \mathrm{V}\right)$. Finally, if $f$ is moreover compactly supported in $\mathrm{B}(0, r) \times \mathrm{V}$ where $\mathrm{B}(0, r)$ is the open ball of radius $r>0$ in $\mathbb{R}_{x}^{d}$ centred in 0 , the function $f_{n}$, for all $n \geq 0$, can be chosen compactly supported in $\mathrm{B}(0, r) \times \mathrm{V}$.

Proof. Let $f \in L^{2}\left(\mathbb{R}_{x}^{d} \times \mathrm{V}\right)$. Let us consider a sequence of mollifier $\left(\rho_{n}\right)_{n \in \mathbb{N}^{*}} \in \mathcal{C}^{\infty}\left(\mathbb{R}_{x}^{d}\right)^{\mathbb{N}^{*}}$, i.e. for all $n \geq 1, \rho_{n}$ is non negative, $\int_{\mathbb{R}_{x}^{d}} \rho_{n}=1$, and $\rho_{n}$ is supported in $\mathrm{B}\left(0,2 n^{-1}\right)$. Define, for all $v \in \mathrm{V}$, the function

$$
x \in \mathbb{R}_{x}^{d} \mapsto f_{n}(x, v)=\int_{\mathbb{R}_{x}^{d}} \rho_{n}(y) f(x-y, v) d y .
$$

For almost every $v \in \mathrm{V}, \int_{\mathbb{R}_{x}^{d}}|f(x, v)|^{2} d x<+\infty$ because $f \in L^{2}\left(\mathbb{R}_{x}^{d} \times \mathrm{V}\right)$. Thus, from 11, Proposition 4.20], for almost every $v \in \mathrm{V}, x \in \mathbb{R}_{x}^{d} \mapsto f_{n}(x, v)$ is $\mathcal{C}^{\infty}$. Using the Young inequality, one has for all $n \geq 1$ and for a.e $v \in \mathrm{V}$ :

$$
\left\|f_{n}(\cdot, v)\right\|_{L^{2}\left(\mathbb{R}_{x}^{d}\right)} \leq\left\|\rho_{n}\right\|_{L^{1}\left(\mathbb{R}_{x}^{d}\right)}\|f(\cdot, v)\|_{L^{2}\left(\mathbb{R}_{x}^{d}\right)}=\|f(\cdot, v)\|_{L^{2}\left(\mathbb{R}_{x}^{d}\right)} \in L^{2}(\mathrm{~V}) .
$$

and thus, $f_{n} \in L^{2}\left(\mathbb{R}_{x}^{d} \times \mathrm{V}\right)$. Since for almost every $v \in \mathrm{V}, f(\cdot, v) \in L^{2}\left(\mathbb{R}_{x}^{d}\right)$, one has from 11 , Theorem 4.22],

$$
\text { for almost every } v \in \mathrm{V}, F_{n}(v)=\left\|f_{n}(\cdot, v)-f(\cdot, v)\right\|_{L^{2}\left(\mathbb{R}_{x}^{d}\right)}^{2} \rightarrow 0 \text { as } n \rightarrow+\infty \text {. }
$$

Since $F_{n}(v) \leq 4\|f(\cdot, v)\|_{L^{2}\left(\mathbb{R}_{x}^{d}\right)}^{2} \in L^{1}(\mathrm{~V})$, the dominated convergence theorem implies that $F_{n} \rightarrow 0$ in $L^{1}(\mathrm{~V})$, i.e. $f_{n} \rightarrow f$ in $L^{2}\left(\mathbb{R}_{x}^{d} \times \mathrm{V}\right)$. In the sense of distribution in $\mathbb{R}_{x}^{d}$, it holds, for all $n \in \mathbb{N}$ and $i \in\{1, \ldots, d\}$,

$$
\partial_{x_{i}} f_{n}=\int_{\mathbb{R}_{x}^{d}} \rho_{n}(y) \partial_{x_{i}} f(x-y, v) d y,
$$

and therefore for almost every $v \in \mathrm{V}$,

$$
v \cdot \partial_{x} f_{n}=\int_{\mathbb{R}_{x}^{d}} \rho_{n}(y)\left[v \cdot \partial_{x} f(x-y, v)\right] d y,
$$

which actually belongs in $L^{2}\left(\mathbb{R}_{x}^{d}\right)$ for almost every $v \in \mathrm{V}$ (because $v \cdot \partial_{x} f \in L^{2}\left(\mathbb{R}_{x}^{d} \times \mathrm{V}\right.$ ) implies that for almost every $v \in \mathrm{V}, v \cdot \partial_{x} f \in L^{2}\left(\mathbb{R}_{x}^{d}\right)$ ). Repeating the previous argument for $v \cdot \partial_{x} f_{n}$ instead of $f_{n}$ and $v \cdot \partial_{x} f$ instead of $f$, we obtain that $v \cdot \partial_{x} f_{n} \in L^{2}\left(\mathbb{R}_{x}^{d} \times \mathrm{V}\right)$ and $v \cdot \partial_{x} f_{n} \rightarrow v \cdot \partial_{x} f$ in $L^{2}\left(\mathbb{R}_{x}^{d} \times \mathrm{V}\right)$ when $n \rightarrow+\infty$. This proves the first claim in Lemma 7 . The second one follows from the fact that, for almost every $v \in \mathrm{V}$, the support of $f_{n}(\cdot, v)$ is included in supp $f(\cdot, v)+\operatorname{supp} \rho_{n}=\operatorname{supp} f(\cdot, v)+\mathrm{B}\left(0,2 n^{-1}\right)$ which is included in $\mathrm{B}(0, r)$ for all $n$ small enough (where, for two subsets $\mathcal{A}$ and $\mathcal{B}$ of $\mathbb{R}^{d}, \mathcal{A}+\mathcal{B}=\{a+b, a \in \mathcal{A}, b \in \mathcal{B}\}$ ). This concludes the proof of the lemma.

Corollary 8. The set $\mathrm{C}_{x}(\mathrm{E})$ is dense in $D\left(\mathrm{P}_{h}\right)$.

Proof. By considering a finite number of open charts covering the compact manifold $\mathrm{M}$ and a partition of unity on $\mathrm{M}$ subordinate to this open cover (with compact supports and indexed by the open cover, which is possible because $M$ is compact), Lemma 7 implies that $C_{x}(E)$ is dense in $D\left(\mathrm{P}_{h}\right)$. 
Let us now end the proof of Proposition 6

Proof of Proposition 6. The operator $\left(\mathrm{P}_{h}, D\left(\mathrm{P}_{h}\right)\right)$ is accretive on $\mathrm{C}_{x}(\mathrm{E})$ (see Lemma 5) and from Corollary 8, it is accretive on $D\left(\mathrm{P}_{h}\right)$. It is clear that the operator $\left(\mathrm{P}_{h}, D\left(\mathrm{P}_{h}\right)\right)$ is closed. The same clearly holds for $\left(\mathrm{P}_{h}^{*}, D\left(\mathrm{P}_{h}\right)\right)$ : it is accretive and closed.

Let us denote by $\left(\mathrm{P}_{h}^{\dagger}, D\left(\mathrm{P}_{h}^{\dagger}\right)\right)$ the adjoint of $\left(\mathrm{P}_{h}, D\left(\mathrm{P}_{h}\right)\right)$ which is defined by:

$$
D\left(\mathrm{P}_{h}^{\dagger}\right)=\left\{f \in L^{2}(\mathrm{E}), \exists g \in L^{2}(\mathrm{E}) \text { s.t } \forall \phi \in D\left(\mathrm{P}_{h}\right),\left\langle\mathrm{P}_{h} \phi, f\right\rangle_{L^{2}(\mathrm{E})}=\langle\phi, g\rangle_{L^{2}(\mathrm{E})}\right\},
$$

and $\mathrm{P}_{h}^{\dagger} f=g$. Let $f \in D\left(\mathrm{P}_{h}^{\dagger}\right)$. Let $\phi \in \mathcal{C}^{\infty}(\mathrm{E})$. Then, one has $\left\langle\mathrm{P}_{h} \phi, f\right\rangle_{L^{2}(\mathrm{E})}=\langle\phi, g\rangle_{L^{2}(\mathrm{E})}$, which implies that the distribution $v \cdot \partial_{x} f$ belongs to $L^{2}(\mathrm{E})$ and thus:

$$
\left\langle\mathrm{P}_{h} \phi, f\right\rangle_{L^{2}(\mathrm{E})}=\left\langle\phi, \mathrm{P}_{h}^{*} f\right\rangle_{L^{2}(\mathrm{E})} .
$$

In particular, for all $\phi \in \mathcal{C}^{\infty}(\mathrm{E}),\langle\phi, g\rangle_{L^{2}(\mathrm{E})}=\left\langle\phi, \mathrm{P}_{h}^{*} f\right\rangle_{L^{2}(\mathrm{E})}$, which leads to $g=\mathrm{P}_{h}^{*} f$. We thus have

$$
\left(\mathrm{P}_{h}^{\dagger}, D\left(\mathrm{P}_{h}^{\dagger}\right)\right) \subset\left(\mathrm{P}_{h}^{*}, D\left(\mathrm{P}_{h}\right)\right) .
$$

Therefore, since $\left(\mathrm{P}_{h}^{*}, D\left(\mathrm{P}_{h}\right)\right)$ is accretive, so is $\left(\mathrm{P}_{h}^{\dagger}, D\left(\mathrm{P}_{h}^{\dagger}\right)\right)$. Because a closed accretive operator with accretive adjoint is $m$-accretive, one deduces that $\mathrm{P}_{h}$ with domain $D\left(\mathrm{P}_{h}\right)$ is $m$-accretive. For the same reasons, $\left(\mathrm{P}_{h}^{\dagger}, D\left(\mathrm{P}_{h}^{\dagger}\right)\right)$ is also $m$-accretive. The same argument shows that $\mathrm{P}_{h}^{*}$ with domain $D\left(\mathrm{P}_{h}\right)$ is also $m$-accretive. Then, Equation (8) implies that,

$$
\left(\mathrm{P}_{h}^{\dagger}, D\left(\mathrm{P}_{h}^{\dagger}\right)\right)=\left(\mathrm{P}_{h}^{*}, D\left(\mathrm{P}_{h}\right)\right) .
$$

It remains to show that $\mathcal{C}^{\infty}(\mathrm{E})$ is a core for both $\mathrm{P}_{h}$ and $\mathrm{P}_{h}^{*}$. Let us denote by $\left(\mathrm{P}_{h}, \mathcal{C}^{\infty}(\mathrm{E})\right)^{\dagger}$ the adjoint of the closable operator $\left(\mathrm{P}_{h}, \mathcal{C}^{\infty}(\mathrm{E})\right)$. On the one hand, by definition of $\left(\mathrm{P}_{h}, \mathcal{C}^{\infty}(\mathrm{E})\right)^{\dagger}$ and reasoning as we proved Equation (8), one has: $\left(\mathrm{P}_{h}, \mathcal{C}^{\infty}(\mathrm{E})\right)^{\dagger} \subset\left(\mathrm{P}_{h}^{*}, D\left(\mathrm{P}_{h}^{*}\right)\right)=\left(\mathrm{P}_{h}^{\dagger}, D\left(\mathrm{P}_{h}^{\dagger}\right)\right)$. Taking the adjoint, leads to

$$
\left(\mathrm{P}_{h}, D\left(\mathrm{P}_{h}\right)\right) \subset \overline{\left(\mathrm{P}_{h}, \mathcal{C}^{\infty}(\mathrm{E})\right)} .
$$

Since the reverse inclusion clearly holds, one gets that $\left(\mathrm{P}_{h}, D\left(\mathrm{P}_{h}\right)\right)=\overline{\left(\mathrm{P}_{h}, \mathcal{C}^{\infty}(\mathrm{E})\right)}$, i.e. $C^{\infty}(\mathrm{E})$ is a core for $\left(\mathrm{P}_{h}, D\left(\mathrm{P}_{h}\right)\right)$. The same holds of course for $\left(\mathrm{P}_{h}^{*}, D\left(\mathrm{P}_{h}\right)\right)$. This concludes the proof of Proposition 6 .

Remark 9. In $[15,27]$, it is shown that when considering the semigroup on $L^{\infty}\left(\mathbb{R}^{d} \times \mathrm{V}\right)$, the space of smooth compactly supported functions on $\mathbb{R}^{d} \times \mathrm{V}$ is a core for $\mathrm{L}_{h}$. Proposition 6 is concerned with the $L^{2}(\mathrm{E})$-setting and also provides a full characterisation of the adjoint of $\mathrm{L}_{h}$, which is required in our analysis.

\section{$3 \quad$ Witten Laplacian associated with $U$ on $\mathrm{M}$}

Our analysis is based on the spectral properties of the Witten Laplacian associated with $U$ on $\mathrm{M}$ in the limit $h \rightarrow 0$. Let us recall that the Witten Laplacian associated with $U$ on $\mathrm{M}$ is the operator

$$
\Delta_{U, h}=-e^{\frac{1}{h} U} h \operatorname{div}_{x} e^{-\frac{1}{h} U} \circ \mathrm{d}_{U, h}=-h^{2} \Delta_{x}+\left|\partial_{x} U\right|^{2}-h \Delta_{x} U,
$$

where $-e^{\frac{1}{h} U} h \operatorname{div}_{x} e^{-\frac{1}{h} U}$ is the formal adjoint of $\mathrm{d}_{U, h}$ in $L^{2}(\mathrm{M})$. The operator $\left(\Delta_{U, h}, H^{2}(\mathrm{M})\right)$ is self-adjoint with compact resolvent on $L^{2}(\mathrm{M})$. Moreover, it is the closure of the Friedrichs extension of the quadratic form

$$
\mathrm{Q}_{U, h}: w \in H^{1}(\mathrm{M}) \mapsto\left\|\mathrm{d}_{U, h} w\right\|_{L^{2}(\mathrm{M})}^{2} .
$$


When (Morse) holds, from 20, 22, there exist $h_{0}>0$ and $\gamma_{0}>0$ such that for all $h \in\left(0, h_{0}\right)$,

$$
\Delta_{U, h} \text { has exactly } \mathrm{m}_{0} \text { eigenvalues smaller than } \gamma_{0} h .
$$

Moreover, these $\mathrm{m}_{0}$ smallest eigenvalues are exponentially small for $h$ small enough, i.e. if $\Phi_{j, h}$ is a $L^{2}(\mathrm{M})$-normalized eigenfunction associated with the $j$-th eigenvalue of $\Delta_{U, h}\left(j \in\left\{1, \ldots, \mathrm{m}_{0}\right\}\right)$, it holds:

$$
\left\|\mathrm{d}_{U, h} \Phi_{j, h}\right\|_{L^{2}(\mathrm{M})} \leq e^{-\frac{c}{h}}
$$

where $c>0$ is a constant independent of $h$. The $\Phi_{j, h}$ 's are two by two orthogonal and from the standard elliptic regularity, for all $j \in\left\{1, \ldots, \mathrm{m}_{0}\right\}, \Phi_{j, h} \in \mathcal{C}^{\infty}(\mathrm{M})$. We have the following lemma which is a direct consequence of the spectral theorem and (9).

Lemma 10. Assume that (Morse) holds. Then, there exists $\delta_{0}>0$ such that for $h$ small enough and for all $w \in H^{2}(\mathrm{M})$ such that $w \in\left\{\Phi_{1, h}, \ldots, \Phi_{\mathrm{m}_{0}, h}\right\}^{\perp_{L^{2}(\mathrm{M})}}$,

$$
\left\langle w,\left[1+\Delta_{U, h}\right]^{-1} \Delta_{U, h} w\right\rangle_{L^{2}(\mathrm{M})} \geq \delta_{0} h\|w\|_{L^{2}(\mathrm{M})}^{2} .
$$

Here and in the following, the space $\left\{\Phi_{1, h}, \ldots, \Phi_{\mathrm{m}_{0}, h}\right\}^{\perp_{L^{2}(\mathrm{M})}}$ denotes the orthogonal of the span of $\left\{\Phi_{1, h}, \ldots, \Phi_{\mathrm{m}_{0}, h}\right\}$ in $L^{2}(\mathrm{M})$. Let us define the vector space

$$
\mathrm{G}=\operatorname{Span}\left(\Phi_{1, h} \mathbf{1}_{\mathrm{V}}, \ldots, \Phi_{\mathrm{m}_{0}, h} \mathbf{1}_{\mathrm{V}}\right),
$$

and the orthogonal projection $\pi_{\mathrm{G}}$ on $\mathrm{G}$ in $L^{2}(\mathrm{E})$, i.e. for all $u \in L^{2}(\mathrm{E})$,

$$
\pi_{\mathrm{G}} u=\sum_{j=1}^{\mathrm{m}_{0}}\left\langle\Phi_{j, h} \mathbf{1}_{\mathrm{V}}, u\right\rangle_{L^{2}(\mathrm{E})} \Phi_{j, h} \mathbf{1}_{\mathrm{V}} .
$$

Let us now give estimates on the terms $\mathrm{P}_{h}\left(\Phi_{j, h} \mathbf{1}_{\mathrm{V}}\right)$ and $\mathrm{P}_{h}^{*}\left(\Phi_{j, h} \mathbf{1}_{\mathrm{V}}\right)$ when $h \rightarrow 0$. Since $\mathrm{P}_{h}\left(\Phi_{j, h} \mathbf{1}_{\mathrm{V}}\right)=-v \cdot \mathrm{d}_{U, h}\left(\Phi_{j, h} \mathbf{1}_{\mathrm{V}}\right)=-v \cdot\left(\mathrm{d}_{U, h} \Phi_{j, h}\right) \mathbf{1}_{\mathrm{V}}$ and $\mathrm{P}_{h}^{*}\left(\Phi_{j, h} \mathbf{1}_{\mathrm{V}}\right)=v \cdot \mathrm{d}_{U, h}\left(\Phi_{j, h}\right) \mathbf{1}_{\mathrm{V}}$, from 10, for all $j \in\left\{1, \ldots, \mathrm{m}_{0}\right\}$ and $h$ small enough, one deduces that:

$$
\left\|\mathrm{P}_{h}\left(\Phi_{j, h} \mathbf{1}_{\mathrm{V}}\right)\right\|_{L^{2}(\mathrm{E})} \leq e^{-\frac{c}{h}} \text { and }\left\|\mathrm{P}_{h}^{*}\left(\Phi_{j, h} \mathbf{1}_{\mathrm{V}}\right)\right\|_{L^{2}(\mathrm{E})} \leq e^{-\frac{c}{h}}
$$

for some $c>0$ independent of $h$. Equation 12 implies that for all $u \in L^{2}(\mathrm{E})$, and $h$ small enough,

$$
\left\|\mathrm{P}_{h} \pi_{\mathrm{G}} u\right\|_{L^{2}(\mathrm{E})} \leq e^{-\frac{c}{h}}\left\|\pi_{\mathrm{G}} u\right\|_{L^{2}(\mathrm{E})} \text { and }\left\|\mathrm{P}_{h}^{*} \pi_{\mathrm{G}} u\right\|_{L^{2}(\mathrm{E})} \leq e^{-\frac{c}{h}}\left\|\pi_{\mathrm{G}} u\right\|_{L^{2}(\mathrm{E})} .
$$

We end this section with an important identity which will be used in the next section. For all $u \in \mathcal{C}^{\infty}(\mathrm{E})$, it holds:

$$
\pi_{v} \Delta_{U, h} \pi_{v} u=\mathrm{m}_{2}^{-1} \pi_{v}\left(v \cdot \mathrm{d}_{U, h}\right)^{*} \circ\left(v \cdot \mathrm{d}_{U, h}\right) \pi_{v} u
$$

where

$$
\mathrm{m}_{2}=\int_{\mathrm{V}} v_{1}^{2} d \nu
$$

and where we have used that $\int_{\vee} v_{i} v_{j} d \nu=0$ for all $i, j \in\{1, \ldots, d\}, i \neq j$ (when $\vee=\mathbb{S}^{d-1}$, this can be proved using the standard polar decomposition, see for instance the proof of 1 , Lemma $36])$.

\section{Resolvent estimates on $\mathrm{P}_{h}$}

Since the computations are exactly the same for $\mathrm{P}_{h}^{\mathrm{BPS}}$ and $\mathrm{P}_{h}^{\mathrm{ZZ}}$, we set, for ease of notation,

$$
\mathrm{P}_{h}=\mathrm{P}_{h}^{\mathrm{BPS}} .
$$

It will be clear from our analysis that the results stated in the remainder of this work also holds for $\mathrm{P}_{h}^{\mathrm{ZZ}}$. 


\subsection{The operator $A_{h}$}

In this section we introduce an operator $A_{h}$ which plays a crucial role in our analysis. Let

$$
\mathrm{T}_{h}=\frac{1}{2}\left(\mathrm{P}_{h}-\mathrm{P}_{h}^{*}\right)=-v \cdot \mathrm{d}_{U, h}+\left(v \cdot \partial_{x} U\right)(\mathrm{I}-\mathrm{B})
$$

be the antisymmetric part of $\mathrm{P}_{h}$, and

$$
\mathrm{S}=\frac{1}{2}\left(\mathrm{P}_{h}+\mathrm{P}_{h}^{*}\right)=\frac{1}{2}\left(\lambda_{1, \mathrm{~J}}+\lambda_{1, \mathrm{~J}}(\cdot,-\cdot)\right)(\mathrm{I}-\mathrm{B})+\lambda_{r}\left(\mathrm{I}-\pi_{v}\right),
$$

be the symmetric part of $\mathrm{P}_{h}$, both with domain $\mathcal{C}^{\infty}(\mathrm{E})$ on $L^{2}(\mathrm{E})$. Notice that $\mathrm{S}$ is independent of $h$. We have the following direct properties:

- The operator $\mathrm{S}$ with domain $\mathcal{C}^{\infty}(\mathrm{E})$ is closable on $L^{2}(\mathrm{E})$ and its closure is a self-adjoint bounded operator on $L^{2}(\mathrm{E})$. Moreover,

$$
\mathrm{S} \pi_{v}=0 .
$$

Furthermore, since $\lambda_{1, \mathrm{~J}} \in L^{\infty}(\mathrm{E})$ and $\lambda_{r} \in L^{\infty}(\mathrm{M})$, there exists $C>0$ such that for all $h$,

$$
\|\mathrm{S}\| \leq C \text {. }
$$

- For all $u \in \mathcal{C}^{\infty}(\mathrm{E}), \mathrm{T}_{h} \pi_{v} u=-v \cdot \mathrm{d}_{U, h} \pi_{v} u$ and consequently, $\left(\mathrm{T}_{h} \pi_{v}\right)^{*} u=-\pi_{v}\left(v \cdot \mathrm{d}_{U, h}\right)^{*} u$, where we recall that $\left(v \cdot \mathrm{d}_{U, h}\right)^{*}=v \cdot\left(-h \partial_{x}+\partial_{x} U\right)$. Therefore, from (14), it holds on $\mathcal{C}^{\infty}(\mathrm{E})$ :

$$
\left(\mathrm{T}_{h} \pi_{v}\right)^{*}\left(\mathrm{~T}_{h} \pi_{v}\right)=\mathrm{m}_{2} \pi_{v} \Delta_{U, h} \pi_{v}=\mathrm{m}_{2} \Delta_{U, h} \pi_{v} .
$$

- For all $u \in \mathcal{C}^{\infty}(\mathrm{E}), \pi_{v} \mathrm{~T}_{h} \pi_{v} u=0$, which follows from $\int_{\mathrm{V}} v_{i} d \nu=0$ for all $i \in\{1, \ldots, d\}$.

Moreover, we have the following result.

Proposition 11. Let $h>0$. It holds as an equality between bounded operators on $L^{2}(\mathrm{E})$,

$$
\left[1+\left(\mathrm{T}_{h} \pi_{v}\right)^{*}\left(\mathrm{~T}_{h} \pi_{v}\right)\right]^{-1} \pi_{v}=\left[1+\mathrm{m}_{2} \Delta_{U, h}\right]^{-1} \pi_{v} .
$$

Let us introduce the operator

$$
\mathrm{A}_{h}=\left[1+\left(\mathrm{T}_{h} \pi_{v}\right)^{*}\left(\mathrm{~T}_{h} \pi_{v}\right)\right]^{-1}\left(\mathrm{~T}_{h} \pi_{v}\right)^{*}=-\left[1+\mathrm{m}_{2} \Delta_{U, h}\right]^{-1} \pi_{v}\left(v \cdot \mathrm{d}_{U, h}\right)^{*}
$$

with domain $\mathcal{C}^{\infty}(\mathrm{E})$ on $L^{2}(\mathrm{E})$. The operator $\mathrm{A}_{h}$ with domain $\mathcal{C}^{\infty}(\mathrm{E})$ is closable on $L^{2}(\mathrm{E})$ and its closure is a bounded operator on $L^{2}(\mathrm{E})$ with norm smaller than 1 . Moreover, for all $u \in \mathcal{C}^{\infty}(\mathrm{E})$, $\pi_{v} \mathrm{~A}_{h} u=\mathrm{A}_{h} u$ and

$$
\left\|\mathrm{T}_{h} \pi_{v} \mathrm{~A}_{h} u\right\|_{L^{2}(\mathrm{E})} \leq\left\|\left(\mathrm{I}-\pi_{v}\right) u\right\|_{L^{2}(\mathrm{E})} .
$$

Finally, one has for all $u \in \mathcal{C}^{\infty}(\mathrm{E}), \mathrm{A}_{h}^{*} u=-v \cdot \mathrm{d}_{U, h}\left[1+\mathrm{m}_{2} \Delta_{U, h}\right]^{-1} \pi_{v} u$, and

$$
\left\|\mathrm{A}_{h}^{*} u\right\|_{L^{2}(\mathrm{E})} \leq \mathrm{m}_{2}^{-1 / 2}\left\|\pi_{v} u\right\|_{L^{2}(\mathrm{E})}
$$

which extends to all $u \in L^{2}(\mathrm{E})$. 
Proof. The proof of Proposition 11 is very similar to those made in [1,14. We recall it in our setting for the sake of completeness.

On the one hand, the operator $\mathrm{T}_{h} \pi_{v}=-v \cdot \mathrm{d}_{U, h} \pi_{v}$ with domain $\mathcal{C}^{\infty}(\mathrm{E})$ is closable and densely defined on $L^{2}(\mathrm{E})$. Thus, from [37, Theorem 5.1.9] (see also [1, Proposition 26]), $1+\left(\mathrm{T}_{h} \pi_{v}\right)^{*}\left(\mathrm{~T}_{h} \pi_{v}\right)$ is a positive self-adjoint operator from $D\left(\left(\mathrm{~T}_{h} \pi_{v}\right)^{*}\left(\mathrm{~T}_{h} \pi_{v}\right)\right)$ to $L^{2}(\mathrm{E})$ and $\left[1+\left(\mathrm{T}_{h} \pi_{v}\right)^{*}\left(\mathrm{~T}_{h} \pi_{v}\right)\right]^{-1}$ is a bounded operator on $L^{2}(\mathrm{E})$. On the other hand, it is standard that $\left[1+\mathrm{m}_{2} \Delta_{U, h}\right]^{-1}$ is a bounded operator on $L^{2}(\mathrm{M})$. Consider $u \in L^{2}(\mathrm{E})$. Let $f_{1} \in L^{2}(\mathrm{E})$ be such that $f_{1}=$ $\left[1+\left(\mathrm{T}_{h} \pi_{v}\right)^{*}\left(\mathrm{~T}_{h} \pi_{v}\right)\right]^{-1} \pi_{v} u$, i.e. $\left[1+\mathrm{m}_{2} \Delta_{U, h} \pi_{v}\right] f_{1}=\pi_{v} u$ (see (17)). Then, one has,

$$
\pi_{v} f_{1}=\pi_{v}^{2} u-\mathrm{m}_{2} \pi_{v} \Delta_{U, h} \pi_{v} f_{1}=\pi_{v} u-\mathrm{m}_{2} \Delta_{U, h} \pi_{v} f_{1}=f_{1} .
$$

Therefore $f_{1}$ is independent of $v \in \mathrm{V}$ and thus, $\left[1+\mathrm{m}_{2} \Delta_{U, h}\right] f_{1}=\pi_{v} u$. This implies that $f_{1}=\left[1+\mathrm{m}_{2} \Delta_{U, h}\right]^{-1} \pi_{v} u$. This proves (18).

Let us now prove the statements concerning $\mathrm{A}_{h}$. To this end, let $u \in \mathcal{C}^{\infty}(\mathrm{E})$. Set

$$
f=\mathrm{A}_{h} u=\left[1+\left(\mathrm{T}_{h} \pi_{v}\right)^{*}\left(\mathrm{~T}_{h} \pi_{v}\right)\right]^{-1}\left(\mathrm{~T}_{h} \pi_{v}\right)^{*} u=-\left[1+\mathrm{m}_{2} \Delta_{U, h}\right]^{-1} \pi_{v}\left(v \cdot \mathrm{d}_{U, h}\right)^{*} u .
$$

Then, it holds: $f=\pi_{v} f \in \mathcal{C}^{\infty}(\mathrm{M})$ (by elliptic regularity). The fact that $\left[1+\left(\mathrm{T}_{h} \pi_{v}\right)^{*}\left(\mathrm{~T}_{h} \pi_{v}\right)\right] f=$ $\left(\mathrm{T}_{h} \pi_{v}\right)^{*} u$ leads to,

$$
\left.\|f\|_{L^{2}(\mathrm{E})}^{2}+\left\|v \cdot \mathrm{d}_{U, h} f\right\|_{L^{2}(\mathrm{E})}^{2}=-\left\langle\pi_{v}\left(v \cdot \mathrm{d}_{U, h}\right)^{*} u\right), f\right\rangle_{L^{2}(\mathrm{E})}=-\left\langle u, v \cdot \mathrm{d}_{U, h} f\right\rangle_{L^{2}(\mathrm{E})} .
$$

Recall that $\pi_{v}\left(v \cdot \mathrm{d}_{U, h} f\right)=\pi_{v} \mathrm{~T}_{h} \pi_{v} f=0$. Consequently $\left\langle u, v \cdot \mathrm{d}_{U, h} f\right\rangle_{L^{2}(\mathrm{E})}=\left\langle\left(\mathbf{I}-\pi_{v}\right) u, v\right.$. $\left.\mathrm{d}_{U, h} f\right\rangle_{L^{2}(\mathrm{E})}$. Using in addition the Cauchy-Schwarz inequality, one deduces that

$$
\max \left(\|f\|_{L^{2}(\mathrm{E})},\left\|v \cdot \mathrm{d}_{U, h} f\right\|_{L^{2}(\mathrm{E})}\right) \leq\left\|\left(\mathrm{I}-\pi_{v}\right) u\right\|_{L^{2}(\mathrm{E})} .
$$

Thus, the closure of $\left(\mathrm{A}_{h}, \mathcal{C}^{\infty}(\mathrm{E})\right)$ exists and is a bounded operator on $L^{2}(\mathrm{E})$ with norm smaller than 1. Moreover, $\left\|v \cdot \mathrm{d}_{U, h} f\right\|_{L^{2}(\mathrm{E})}=\left\|\left(\mathrm{T}_{h} \pi_{v}\right) f\right\|_{L^{2}(\mathrm{E})} \leq\left\|\left(\mathrm{I}-\pi_{v}\right) u\right\|_{L^{2}(\mathrm{E})}$, which proves 199.

Let us now prove the statements concerning $A_{h}^{*}$. It is clear, using integration by parts, that for all $u \in \mathcal{C}^{\infty}(\mathrm{E}), \mathrm{A}_{h}^{*} u=-v \cdot \mathrm{d}_{U, h}\left[1+\mathrm{m}_{2} \Delta_{U, h}\right]^{-1} \pi_{v} u$. Set $f=\left[1+\mathrm{m}_{2} \Delta_{U, h}\right]^{-1} \pi_{v} u$ which is a function independent of $v \in \mathrm{V}$ and belongs to $\mathcal{C}^{\infty}(\mathrm{E})$. It thus holds $\mathrm{A}_{h}^{*} u=-v \cdot \mathrm{d}_{U, h} f$ and $\left\|\mathrm{A}_{h}^{*} u\right\|_{L^{2}(\mathrm{E})}=\left\|v \cdot \mathrm{d}_{U, h} f\right\|_{L^{2}(\mathrm{E})} \leq\left\|\mathrm{d}_{U, h} f\right\|_{L^{2}(\mathrm{M})}$. The function $f$ is solution to

$$
f+\mathrm{m}_{2} \Delta_{U, h} f=\pi_{v} u \text { on } \mathrm{M} .
$$

This implies that

$$
\|f\|_{L^{2}(\mathrm{M})}^{2}+\mathrm{m}_{2}\left\|\mathrm{~d}_{U, h} f\right\|_{L^{2}(\mathrm{M})}^{2} \leq\left|\left\langle\pi_{v} u, f\right\rangle_{L^{2}(\mathrm{M})}\right|,
$$

and therefore, $\|f\|_{L^{2}(\mathrm{M})} \leq\left\|\pi_{v} u\right\|_{L^{2}(\mathrm{M})}$ and $\left\|\mathrm{d}_{U, h} f\right\|_{L^{2}(\mathrm{M})} \leq \mathrm{m}_{2}^{-1 / 2}\left\|\pi_{v} u\right\|_{L^{2}(\mathrm{M})}$. This proves (20) and concludes the proof of Proposition 11 .

The operator $A_{h}$ is the corner stone of our analysis. This operator was used in [1, 14 to prove the convergence to the equilibrium measure when $h>0$ is fixed. A similar operator was introduced in 21, 24, 39] to study the Boltzmann equation and the Kramers-Fokker-Planck equation. We also refer to [26] in this connection. 


\subsection{Semi-classical hypocoercive estimate for $\mathrm{P}_{h}$}

The aim of this section is to prove Proposition 12 which gives a semiclassical hypocoercive estimate on $\mathrm{P}_{h}$. Such estimates were first derived in 24 (see also [39]) for the Boltzmann operator.

Proposition 12. Let us assume that (Morse) holds. There exist $c_{0}>0$ and $h_{0}>0$ such that for all $h \in\left(0, h_{0}\right)$ and all for all $u \in \mathcal{C}^{\infty}(\mathrm{E}) \cap \mathrm{G}^{\perp_{L^{2}}(\mathrm{E})}$, it holds:

$$
\operatorname{Re}\left\langle\mathrm{P}_{h} u,\left[1+\varepsilon_{0} h\left(\mathrm{~A}_{h}+\mathrm{A}_{h}^{*}\right)\right] u\right\rangle_{L^{2}(\mathrm{E})} \geq c_{0} h^{2}\|u\|_{L^{2}(\mathrm{E})}^{2} .
$$

This implies that for all $c_{1} \in\left(0, c_{0}\right)$, if $\operatorname{Re} z \leq \frac{c_{1}}{2} h^{2}$, there exists $h_{0}>0$ such that for all $h \in\left(0, h_{0}\right)$ and for all $u \in \mathcal{C}^{\infty}(\mathrm{E}) \cap \mathrm{G}^{\perp_{L^{2}}(\mathrm{E})}$ :

$$
\left\|\left(\mathrm{P}_{h}-z\right) u\right\|_{L^{2}(\mathrm{E})} \geq \frac{c_{1}}{2} h^{2}\|u\|_{L^{2}(\mathrm{E})} .
$$

This equality extends to all $u \in D\left(\mathrm{P}_{h}\right) \cap \mathrm{G}^{\perp^{2}(\mathrm{E})}$. Finally, Equation (23) holds for $\mathrm{P}_{h}^{*}$ (with different constants).

Here and in the following, the space $\mathrm{G}^{{ }^{2}}{ }^{2}(\mathrm{E})$ denotes the orthogonal of $\mathrm{G}$ in $L^{2}(\mathrm{E})$.

Let us prove Proposition 12

Proof of Proposition 12. Let $\varepsilon>0$. For $u \in \mathcal{C}^{\infty}(\mathrm{E})$, one has from Lemma 5 and (2):

$$
\begin{aligned}
\operatorname{Re}\left\langle\mathrm{P}_{h} u,\left[1+\varepsilon\left(\mathrm{A}_{h}+\mathrm{A}_{h}^{*}\right)\right] u\right\rangle_{L^{2}(\mathrm{E})} & =\operatorname{Re}\left\langle\mathrm{P}_{h} u, u\right\rangle_{L^{2}(\mathrm{E})}+\varepsilon \operatorname{Re}\left\langle\mathrm{P}_{h} u,\left(\mathrm{~A}_{h}+\mathrm{A}_{h}^{*}\right) u\right\rangle_{L^{2}(\mathrm{E})} \\
& \geq r_{0}\left\|\left(\mathrm{I}-\pi_{v}\right) f\right\|_{L^{2}(\mathrm{E})}^{2}+\varepsilon \operatorname{Re}\left\langle\mathrm{P}_{h} u,\left(\mathrm{~A}_{h}+\mathrm{A}_{h}^{*}\right) u\right\rangle_{L^{2}(\mathrm{E})} .
\end{aligned}
$$

In addition,

$$
\begin{aligned}
\operatorname{Re}\left\langle\mathrm{P}_{h} u,\left(\mathrm{~A}_{h}+\mathrm{A}_{h}^{*}\right) u\right\rangle_{L^{2}(\mathrm{E})}= & \operatorname{Re}\left\langle\mathrm{S} u,\left(\mathrm{~A}_{h}+\mathrm{A}_{h}^{*}\right) u\right\rangle_{L^{2}(\mathrm{E})}+\operatorname{Re}\left\langle\mathrm{T}_{h} u,\left(\mathrm{~A}_{h}+\mathrm{A}_{h}^{*}\right) u\right\rangle_{L^{2}(\mathrm{E})} \\
= & \operatorname{Re}\left\langle\mathrm{A}_{h} \mathrm{~S} u, u\right\rangle_{L^{2}(\mathrm{E})}+\operatorname{Re}\left\langle\mathrm{A}_{h} \mathrm{~T}_{h}\left(\mathrm{I}-\pi_{v}\right) u, u\right\rangle_{L^{2}(\mathrm{E})} \\
& +\operatorname{Re}\left\langle\mathrm{S} u, \mathrm{~A}_{h} u\right\rangle_{L^{2}(\mathrm{E})}+\operatorname{Re}\left\langle\mathrm{T}_{h} u, \mathrm{~A}_{h} u\right\rangle_{L^{2}(\mathrm{E})} \\
& +\operatorname{Re}\left\langle\mathrm{A}_{h} \mathrm{~T}_{h} \pi_{v} u, u\right\rangle_{L^{2}(\mathrm{E})}
\end{aligned}
$$

We will now estimate each of the above terms. In the following $C>0$ is a constant independent of $h$ and $u$ which can change from one occurrence to another.

Step 1. Lower bound on $\varepsilon \operatorname{Re}\left\langle\mathrm{A}_{h} \mathrm{~T}_{h} \pi_{v} u, u\right\rangle_{L^{2}(\mathrm{E})}$. Because $\pi_{v} \mathrm{~A}_{h}=\mathrm{A}_{h}$ (see Proposition 11) it holds

$$
\operatorname{Re}\left\langle\mathrm{A}_{h} \mathrm{~T}_{h} \pi_{v} u, u\right\rangle_{L^{2}(\mathrm{E})}=\operatorname{Re}\left\langle\mathrm{A}_{h} \mathrm{~T}_{h} \pi_{v} u, \pi_{v} u\right\rangle_{L^{2}(\mathrm{E})} .
$$

From (17) and (18), we recall that:

$$
\mathrm{A}_{h} \mathrm{~T}_{h} \pi_{v}=\left[1+\mathrm{m}_{2} \Delta_{U, h}\right]^{-1} \mathrm{~m}_{2} \Delta_{U, h} \pi_{v} \text { on } \mathcal{C}^{\infty}(\mathrm{E}),
$$

Assume that (Morse) holds. From Lemma 10, there exists $\delta_{0}>0$ such that for $h$ small enough and for all $w \in \mathcal{C}^{\infty}(\mathrm{M}) \cap\left\{\Phi_{1, h}, \ldots, \Phi_{\mathrm{m}_{0}, h}\right\}^{\perp_{L^{2}(\mathrm{M})}}$,

$$
\left\langle\mathrm{m}_{2}\left[1+\mathrm{m}_{2} \Delta_{U, h}\right]^{-1} \Delta_{U, h} w, w\right\rangle_{L^{2}(\mathrm{M})} \geq \delta_{0} h\|w\|_{L^{2}(\mathrm{M})}^{2} .
$$

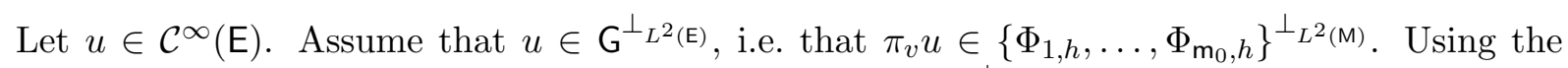
previous inequality, one deduces that for all $u \in \mathcal{C}^{\infty}(\mathrm{E}) \cap \mathrm{G}^{\perp} L^{2}(\mathrm{E})$ :

$$
\varepsilon \operatorname{Re}\left\langle\mathrm{A}_{h} \mathrm{\top}_{h} \pi_{v} u, u\right\rangle_{L^{2}(\mathrm{E})}=\varepsilon \operatorname{Re}\left\langle\mathrm{A}_{h} \mathrm{~T}_{h} \pi_{v} u, \pi_{v} u\right\rangle_{L^{2}(\mathrm{E})} \geq \varepsilon \delta_{0} h\left\|\pi_{v} u\right\|_{L^{2}(\mathrm{E})}^{2} .
$$


Step 2. Upper bound on the term $\operatorname{Re}\left\langle\mathrm{A}_{h} \mathrm{~S} u, u\right\rangle_{L^{2}(\mathrm{E})}+\operatorname{Re}\left\langle\mathrm{A}_{h} \mathrm{~T}_{h}\left(\mathrm{I}-\pi_{v}\right) u, u\right\rangle_{L^{2}(\mathrm{E})}$.

Let $u \in \mathcal{C}^{\infty}(\mathrm{E})$. Using (15), one has: $\left\langle\mathrm{A}_{h} \mathrm{~S} u, u\right\rangle_{L^{2}(\mathrm{E})}=\left\langle\left(\mathrm{I}-\pi_{v}\right) u, \mathrm{SA}_{h}^{*} u\right\rangle_{L^{2}(\mathrm{E})}$. Thus from 16), one has for all $u \in \mathcal{C}^{\infty}(\mathrm{E})$,

$$
\left\langle\mathrm{A}_{h} \mathrm{~S} u, u\right\rangle_{L^{2}(\mathrm{E})} \leq C\left\|\left(\mathrm{I}-\pi_{v}\right) u\right\|_{L^{2}(\mathrm{E})}\|\mathrm{S}\|\left\|\mathrm{A}_{h}^{*} u\right\|_{L^{2}(\mathrm{E})} \leq C\left\|\left(\mathrm{I}-\pi_{v}\right) u\right\|_{L^{2}(\mathrm{E})}\left\|\mathrm{A}_{h}^{*} u\right\|_{L^{2}(\mathrm{E})} .
$$

Using $(20)$, one deduces that for all $u \in \mathcal{C}^{\infty}(\mathrm{E})$,

$$
\left\langle\mathrm{A}_{h} \mathrm{~S} u, u\right\rangle_{L^{2}(\mathrm{E})} \leq C\left\|\left(\mathrm{I}-\pi_{v}\right) u\right\|_{L^{2}(\mathrm{E})}\left\|\pi_{v} u\right\|_{L^{2}(\mathrm{E})} .
$$

Let us now deal with the term $\left\langle\mathrm{A}_{h} \mathrm{~T}_{h}\left(\mathrm{I}-\pi_{v}\right) u, u\right\rangle_{L^{2}(\mathrm{E})}$. One has for all $u \in \mathcal{C}^{\infty}(\mathrm{E})$,

$$
\left\langle\mathrm{A}_{h} \mathrm{~T}_{h}\left(\mathrm{I}-\pi_{v}\right) u, u\right\rangle_{L^{2}(\mathrm{E})}=-\left\langle\left(\mathrm{I}-\pi_{v}\right) u, \mathrm{~T}_{h} \mathrm{~A}_{h}^{*} u\right\rangle_{L^{2}(\mathrm{E})} \leq\left\|\left(\mathrm{I}-\pi_{v}\right) u\right\|_{L^{2}(\mathrm{E})}\left\|\mathrm{T}_{h} \mathrm{~A}_{h}^{*} u\right\|_{L^{2}(\mathrm{E})},
$$

where we used that $\mathrm{T}_{h}^{*}=-\mathrm{T}_{h}$ on $\mathcal{C}^{\infty}(\mathrm{E})$ and $\mathrm{A}_{h}^{*} u \in \mathcal{C}^{\infty}(\mathrm{E})$ by elliptic regularity. Let $f=$ $\left[1+\mathrm{m}_{2} \Delta_{U, h}\right]^{-1} \pi_{v} u$. Then, it holds:

$$
\begin{aligned}
\mathrm{T}_{h} \mathrm{~A}_{h}^{*} u & =\left[v \cdot \mathrm{d}_{U, h}-\left(v \cdot \partial_{x} U\right)(\mathrm{I}-\mathrm{B})\right] v \cdot \mathrm{d}_{U, h} f \\
& =\left[v \cdot h \partial_{x}+v \cdot \partial_{x} U-\left(v \cdot \partial_{x} U\right)(\mathrm{I}-\mathrm{B})\right] v \cdot \mathrm{d}_{U, h} f \\
& =v \cdot h \partial_{x}\left(v \cdot h \partial_{x} f+f v \cdot \partial_{x} U\right)+\left[v \cdot \partial_{x} U-\left(v \cdot \partial_{x} U\right)(\mathrm{I}-\mathrm{B})\right] v \cdot \mathrm{d}_{U, h} f .
\end{aligned}
$$

Therefore, since $v \cdot \partial_{x} U$ is a bounded operator on $L^{2}(\mathrm{E})$, for $h$ small enough, it holds:

$$
C\left\|\mathrm{~T}_{h} \mathrm{~A}_{h}^{*} u\right\|_{L^{2}(\mathrm{E})} \leq h^{2}\|f\|_{H^{2}(\mathrm{M})}+h\left\|\partial_{x} f\right\|_{L^{2}(\mathrm{M})}+\|f\|_{L^{2}(\mathrm{M})} .
$$

From (21) and the lines after, one has,

$$
\|f\|_{L^{2}(\mathrm{M})}+\left\|\mathrm{d}_{U, h} f\right\|_{L^{2}(\mathrm{M})} \leq C\left\|\pi_{v} u\right\|_{L^{2}(\mathrm{M})} .
$$

Moreover, Equation (21) writes

$$
-\mathrm{m}_{2} h^{2} \Delta_{x} f=\mathrm{m}_{2}\left(h \Delta_{x} U-\left|\partial_{x} U\right|^{2}\right) f-f+\pi_{v} u .
$$

Thus, multiplying by $f$ and using integration by parts, for all $h$ such that $|h| \leq 1$, it holds:

$$
h^{2}\left\|\partial_{x} f\right\|_{L^{2}(\mathrm{M})}^{2} \leq C\left\|\pi_{v} u\right\|_{L^{2}(\mathrm{M})}^{2} .
$$

Let us recall that from the standard elliptic regularity on $\mathrm{M}$, one has,

$$
\|f\|_{H^{2}(\mathrm{M})} \leq C\left(\left\|\Delta_{x} f\right\|_{L^{2}(\mathrm{M})}+\|f\|_{L^{2}(\mathrm{M})}\right) .
$$

Thus for $|h| \leq 1$ small enough, it holds:

$$
\left.\|f\|_{H^{2}(\mathrm{M})} \leq C\left[h^{-2}\left(\|f\|_{L^{2}(\mathrm{M})}+\left\|\pi_{v} u\right\|_{L^{2}(\mathrm{M})}\right)+\|f\|_{L^{2}(\mathrm{M})}\right)\right] \leq C h^{-2}\left\|\pi_{v} u\right\|_{L^{2}(\mathrm{M})} .
$$

In conclusion, for all $u \in \mathcal{C}^{\infty}(\mathrm{E})$,

$$
\left\langle\mathrm{A}_{h} \mathrm{~T}_{h} u, u\right\rangle_{L^{2}(\mathrm{E})} \leq C\left\|\left(\mathrm{I}-\pi_{v}\right) u\right\|_{L^{2}(\mathrm{E})}\left\|\pi_{v} u\right\|_{L^{2}(\mathrm{E})} .
$$

Step 3. Upper bound on the term $\operatorname{Re}\left\langle\mathrm{S} u, \mathrm{~A}_{h} u\right\rangle_{L^{2}(\mathrm{E})}+\operatorname{Re}\left\langle\mathrm{T}_{h} u, \mathrm{~A}_{h} u\right\rangle_{L^{2}(\mathrm{E})}$.

On the one hand, from Proposition 11, it holds $\pi_{v} \mathrm{~A}_{h}=\mathrm{A}_{h}$, and since from (15), $\pi_{v} \mathrm{~S}=0$, one has, for all $u \in \mathcal{C}^{\infty}(\mathrm{E})$,

$$
\left\langle\mathrm{S} u, \mathrm{~A}_{h} u\right\rangle_{L^{2}(\mathrm{E})}=\left\langle\pi_{v} \mathrm{~S} u, \mathrm{~A}_{h} u\right\rangle_{L^{2}(\mathrm{E})}=0 .
$$


On the other hand, since $\pi_{v} \mathrm{~T}_{h} \pi_{v}=0$, one has, for all $u \in \mathcal{C}^{\infty}(\mathrm{E})$,

$$
\begin{aligned}
\left\langle\mathrm{T}_{h} u, \mathrm{~A}_{h} u\right\rangle_{L^{2}(\mathrm{E})} & =\left\langle\mathrm{T}_{h} u, \pi_{v} \mathrm{~A}_{h} u\right\rangle_{L^{2}(\mathrm{E})} \\
& =\left\langle\pi_{v} \mathrm{~T}_{h}\left(\mathrm{I}-\pi_{v}\right) u, \pi_{v} \mathrm{~A}_{h} u\right\rangle_{L^{2}(\mathrm{E})} \\
& =-\left\langle\left(\mathrm{I}-\pi_{v}\right) u, \mathrm{~T}_{h} \pi_{v} \mathrm{~A}_{h} u\right\rangle_{L^{2}(\mathrm{E})} \leq\left\|\left(\mathrm{I}-\pi_{v}\right) u\right\|_{L^{2}(\mathrm{E})}\left\|\mathrm{T}_{h} \pi_{v} \mathrm{~A}_{h}\right\|_{L^{2}(\mathrm{E})} .
\end{aligned}
$$

Since $\left\|\mathrm{T}_{h} \pi_{v} \mathrm{~A}_{h}\right\|_{L^{2}(\mathrm{E})} \leq\left\|\left(\mathrm{I}-\pi_{v}\right) u\right\|_{L^{2}(\mathrm{E})}$ (see (19)), one deduces that for all $u \in \mathcal{C}^{\infty}(\mathrm{E})$,

$$
\left\langle\mathrm{T}_{h} u, \mathrm{~A}_{h} u\right\rangle_{L^{2}(\mathrm{E})} \leq C\left\|\left(\mathrm{I}-\pi_{v}\right) u\right\|_{L^{2}(\mathrm{E})}^{2} .
$$

Step 4. We have proved that there exist $C>0$ and $\delta_{0}>0$ such that for all $u \in \mathcal{C}^{\infty}(\mathrm{E}) \cap \mathrm{G}^{\perp_{L^{2}(\mathrm{E})}}$ and $h$ small enough, it holds:

$$
\begin{aligned}
\operatorname{Re}\left\langle\mathrm{P}_{h} u,\left[1+\varepsilon\left(\mathrm{A}_{h}+\mathrm{A}_{h}^{*}\right)\right] u\right\rangle_{L^{2}(\mathrm{E})} \geq & r_{0}\left\|\left(\mathrm{I}-\pi_{v}\right) u\right\|_{L^{2}(\mathrm{E})}^{2}+\varepsilon \delta_{0} h\left\|\pi_{v} u\right\|_{L^{2}(\mathrm{E})}^{2} \\
& -C \varepsilon\left\|\left(\mathbf{I}-\pi_{v}\right) u\right\|_{L^{2}(\mathrm{E})}\left\|\pi_{v} u\right\|_{L^{2}(\mathrm{E})} \\
& -C \varepsilon\left\|\left(\mathbf{I}-\pi_{v}\right) u\right\|_{L^{2}(\mathrm{E})}^{2} .
\end{aligned}
$$

Let $\lambda_{0}=\delta_{0} / C$. Define $\lambda_{v}=r_{0} / C$ and $\lambda_{x}(h)=\lambda_{0} h$. Then for $h$ small enough, one has

$$
\begin{aligned}
C^{-1} \operatorname{Re}\left\langle\mathrm{P}_{h} u,\left[1+\varepsilon\left(\mathrm{A}_{h}+\mathrm{A}_{h}^{*}\right)\right] u\right\rangle_{L^{2}(\mathrm{E})} \geq & \left(\lambda_{v}-\varepsilon\right)\left\|\left(\mathrm{I}-\pi_{v}\right) u\right\|_{L^{2}(\mathrm{E})}^{2}+\varepsilon \lambda_{x}(h)\left\|\pi_{v} u\right\|_{L^{2}(\mathrm{E})}^{2} \\
& -\varepsilon\left\|\left(\mathrm{I}-\pi_{v}\right) u\right\|_{L^{2}(\mathrm{E})}\left\|\pi_{v} u\right\|_{L^{2}(\mathrm{E})} \\
= & X_{h}(u)^{t} M_{h} X_{h}(u),
\end{aligned}
$$

where the vector field $X_{h}(u)$ equals to

$$
X_{h}(u)=\left(\left\|\pi_{v} u\right\|_{L^{2}(\mathrm{E})},\left\|\left(\mathrm{I}-\pi_{v}\right) u\right\|_{L^{2}(\mathrm{E})}\right)^{t},
$$

and the symmetric $2 \times 2$ real matrix $M_{h}$ equals to:

$$
M_{h}=\left(\begin{array}{cc}
\varepsilon \lambda_{x}(h) & -\frac{\varepsilon}{2} \\
-\frac{\varepsilon}{2} & \lambda_{v}-\varepsilon
\end{array}\right) .
$$

According to [1, Section 3.1 and Lemma 23] (here $\lambda_{x}(h)<1$ for $h$ small enough), for all $h$ small enough, the smallest eigenvalue $\Lambda_{0}(\varepsilon)$ of $M_{h}$ is non negative providing that:

$$
\varepsilon \leq \frac{4 \lambda_{v} \lambda_{x}(h)}{4 \lambda_{x}(h)+1}
$$

and equals

$$
2 \Lambda_{0}(\varepsilon)=\lambda_{v}-\varepsilon\left(1-\lambda_{x}(h)\right)-\sqrt{\left[\lambda_{v}-\varepsilon\left(1-\lambda_{x}(h)\right)\right]^{2}-4 \varepsilon \lambda_{x}(h)\left(\lambda_{v}-\varepsilon\right)+\varepsilon^{2}} .
$$

When $h>0$ fixed, from [1, item (b) Lemma 24], $\varepsilon \in\left[0,4 \lambda_{x}(h) \lambda_{v} /\left(4 \lambda_{x}(h)+1\right)\right] \mapsto \Lambda_{0}(\varepsilon)$ (see [1, item (b) Lemma 24]) reaches its maximum at a unique point $\varepsilon_{\max }$ whose expression is given by [1, Lemma 23$]^{2}$,

$$
\varepsilon_{\max }=\lambda_{v} \times \frac{1+\lambda_{x}(h)-\left(1-\lambda_{x}(h)\right)\left[1 /\left(1+4 \lambda_{x}(h)\right)\right]^{\frac{1}{2}}}{\left(1+\lambda_{x}(h)\right)^{2}+1} .
$$

\footnotetext{
${ }^{2}$ This follows from the fact that, with the notation of $\left[1, \Lambda_{0}(\varepsilon)=\lambda_{v} \Lambda\left(\varepsilon / \lambda_{v}\right)\right.$.
} 
An asymptotic expansion when $h \rightarrow 0$ of $\varepsilon_{\max }$ then leads to $\varepsilon_{\max }=\varepsilon_{0} h+O\left(h^{2}\right)$ where $\varepsilon_{0}=\lambda_{v} \lambda_{0}$. Then, one has in the limit $h \rightarrow 0$ :

$$
2 \Lambda_{0}\left(\varepsilon_{0} h\right)=2 \varepsilon_{0} \lambda_{0}\left(1-\frac{\varepsilon_{0}}{4 \lambda_{v} \lambda_{0}}\right) h^{2}+O\left(h^{3}\right)=\frac{3}{2} \lambda_{v} \lambda_{0}^{2} h^{2}+O\left(h^{3}\right) .
$$

Indeed, one has:

$$
2 \Lambda_{0}\left(\varepsilon_{0} h\right)=\lambda_{v}-\varepsilon_{0} h+\varepsilon_{0} \lambda_{0} h^{2}-\sqrt{\lambda_{v}^{2}-2 \varepsilon_{0} \lambda_{v} h+\mathrm{p} h^{2}+O\left(h^{3}\right)},
$$

with $\mathrm{p}=2 \varepsilon_{0} \lambda_{0} \lambda_{v}+\varepsilon_{0}^{2}-4 \varepsilon_{0} \lambda_{0} \lambda_{v}+\varepsilon_{0}^{2}=-2 \varepsilon_{0} \lambda_{0} \lambda_{v}+2 \varepsilon_{0}^{2}$. Then, when $h \rightarrow 0$,

$$
\begin{aligned}
-\lambda_{v} \sqrt{1-\frac{2 \varepsilon_{0}}{\lambda_{v}} h+\frac{\mathrm{p}}{\lambda_{v}^{2}} h^{2}+O\left(h^{3}\right)} & =-\lambda_{v}\left[1+\frac{1}{2}\left(-\frac{2 \varepsilon_{0}}{\lambda_{v}} h+\frac{\mathrm{p}}{\lambda_{v}^{2}} h^{2}\right)-\frac{1}{8} \frac{4 \varepsilon_{0}^{2}}{\lambda_{v}^{2}} h^{2}+O\left(h^{3}\right)\right] \\
& =-\lambda_{v}+\varepsilon_{0} h+\left(-\frac{\mathrm{p}}{2 \lambda_{v}}+\frac{\varepsilon_{0}^{2}}{2 \lambda_{v}^{2}}\right) h^{2}+O\left(h^{3}\right) .
\end{aligned}
$$

This implies that

$$
2 \Lambda_{0}\left(\varepsilon_{0} h\right)=\left[\varepsilon_{0} \lambda_{0}-\frac{\mathrm{p}}{2 \lambda_{v}}+\frac{\varepsilon_{0}^{2}}{2 \lambda_{v}}\right] h^{2}+O\left(h^{3}\right)=\left(2 \varepsilon_{0} \lambda_{0}-\frac{\varepsilon_{0}^{2}}{2 \lambda_{v}}\right) h^{2}+O\left(h^{3}\right),
$$

which is $(27)$. Thus, there exists $c_{0}>0$ such that for $h$ small enough and for all $u \in \mathcal{C}^{\infty}(\mathrm{E}) \cap$ $\mathrm{G}^{\perp} L^{2}(\mathrm{E})$, one has:

$$
\operatorname{Re}\left\langle\mathrm{P}_{h} u,\left[1+\varepsilon_{0} h\left(\mathrm{~A}_{h}+\mathrm{A}_{h}^{*}\right)\right] u\right\rangle_{L^{2}(\mathrm{E})} \geq c_{0} h^{2}\|u\|_{L^{2}(\mathrm{E})}^{2} .
$$

This concludes the proof of the first statement in Proposition 12, namely 222). Let us now prove $(23)$. Let $z \in \mathbb{C}$ and $u \in \mathcal{C}^{\infty}(\mathrm{E}) \cap \mathrm{G}^{\perp^{2}(\mathrm{E})}$. Then, one has:

$\left\|\left(\mathrm{P}_{h}-z\right) u\right\|_{L^{2}(\mathrm{E})}\left\|\left(1+\varepsilon_{0} h\left(A_{h}+\mathrm{A}_{h}^{*}\right)\right) u\right\|_{L^{2}(\mathrm{E})} \geq c_{0} h^{2}\|u\|_{L^{2}(\mathrm{E})}^{2}-\operatorname{Re}\left(z\left\langle u,\left[1+\varepsilon_{0} h\left(\mathrm{~A}_{h}+\mathrm{A}_{h}^{*}\right)\right] u\right\rangle_{L^{2}(\mathrm{E})}\right)$.

Since $1+\varepsilon_{0} h\left(\mathrm{~A}_{h}+\mathrm{A}_{h}^{*}\right)$ is a (bounded) self-adjoint operator, it holds $\left\langle u,\left[1+\varepsilon_{0} h\left(\mathrm{~A}_{h}+\mathrm{A}_{h}^{*}\right)\right] u\right\rangle_{L^{2}(\mathrm{E})} \in$ $\mathbb{R}$. Moreover, for all $w \in \mathcal{C}^{\infty}(\mathrm{E})$ :

$$
\begin{aligned}
\left\langle w,\left[1+\varepsilon_{0} h\left(\mathrm{~A}_{h}+\mathrm{A}_{h}^{*}\right)\right] w\right\rangle_{L^{2}(\mathrm{E})} & =\|w\|_{L^{2}(\mathrm{E})}^{2}+\varepsilon_{0} h\left\langle w,\left(\mathrm{~A}_{h}+\mathrm{A}_{h}^{*}\right) w\right\rangle_{L^{2}(\mathrm{E})} \\
& \geq\|w\|_{L^{2}(\mathrm{E})}^{2}-\varepsilon_{0} h\|w\|_{L^{2}(\mathrm{E})}^{2}\left(\left\|\mathrm{~A}_{h}\right\|+\left\|\mathrm{A}_{h}^{*}\right\|\right) \\
& \geq\left(1-2 \varepsilon_{0} h\right)\|w\|_{L^{2}(\mathrm{E})}^{2} \geq 0,
\end{aligned}
$$

for $h>0$ such that $2 \varepsilon_{0} h<1$ and where we have used that $\left\|\mathrm{A}_{h}^{*}\right\|=\left\|\mathrm{A}_{h}\right\| \leq 1$, see Proposition 11 . Thus, one has:

$$
0 \leq\left\langle w,\left[1+\varepsilon_{0} h\left(\mathrm{~A}_{h}+\mathrm{A}_{h}^{*}\right)\right] w\right\rangle_{L^{2}(\mathrm{E})} \leq\|w\|_{L^{2}(\mathrm{E})}\left\|\left[1+\varepsilon_{0} h\left(\mathrm{~A}_{h}+\mathrm{A}_{h}^{*}\right)\right] w\right\|_{L^{2}(\mathrm{E})} .
$$

Thus, for all $u \in \mathcal{C}^{\infty}(\mathrm{E}) \cap \mathrm{G}^{\perp^{2}(\mathrm{E})}$, one deduces that:

$$
\begin{aligned}
\left\|\left(\mathrm{P}_{h}-z\right) u\right\|_{L^{2}(\mathrm{E})}\left\|\left(1+\varepsilon_{0} h\left(\mathrm{~A}_{h}+\mathrm{A}_{h}^{*}\right)\right) u\right\|_{L^{2}(\mathrm{E})} \geq & c_{0} h^{2}\|u\|_{L^{2}(\mathrm{E})}^{2} \\
& -(\operatorname{Re} z)_{+}\|u\|_{L^{2}(\mathrm{E})}\left\|\left[1+\varepsilon_{0} h\left(\mathrm{~A}_{h}+\mathrm{A}_{h}^{*}\right)\right] u\right\|_{L^{2}(\mathrm{E})},
\end{aligned}
$$

where we recall that $(\operatorname{Re} z)_{+}=\max (\operatorname{Re} z, 0)$. Consequently, one has:

$$
\left\|\left(\mathrm{P}_{h}-z\right) u\right\|_{L^{2}(\mathrm{E})}\left[1+\varepsilon_{0} h\left(\left\|\mathrm{~A}_{h}\right\|+\left\|\mathrm{A}_{h}^{*}\right\|\right)\right]\|u\|_{L^{2}(\mathrm{E})} \geq\left[c_{0} h^{2}-(\operatorname{Re} z)_{+}\left[1+\varepsilon_{0} h\left(\left\|\mathrm{~A}_{h}\right\|+\left\|\mathrm{A}_{h}^{*}\right\|\right)\right]\right]\|u\|_{L^{2}(\mathrm{E})}^{2},
$$


and therefore,

$$
\left\|\left(\mathrm{P}_{h}-z\right) u\right\|_{L^{2}(\mathrm{E})} \geq\left[\frac{c_{0} h^{2}}{1+2 \varepsilon_{0} h}-(\operatorname{Re} z)_{+}\right]\|u\|_{L^{2}(\mathrm{E})} .
$$

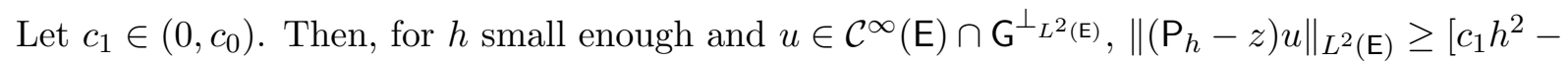
$\left.(\operatorname{Re} z)_{+}\right]\|u\|_{L^{2}(\mathrm{E})}^{2}$. Let us assume that $\operatorname{Re} z \leq \frac{c_{1}}{2} h^{2}$. Then, $\left\|\left(\mathrm{P}_{h}-z\right) u\right\|_{L^{2}(\mathrm{E})} \geq \frac{c_{1}}{2} h^{2}\|u\|_{L^{2}(\mathrm{E})}^{2}$. This proves (23).

Let us show that this equality extends to all $u \in D\left(\mathrm{P}_{h}\right) \cap \mathrm{G}^{L_{L}{ }^{2}(\mathrm{E})}$. Let $u \in D\left(\mathrm{P}_{h}\right) \cap \mathrm{G}^{\perp_{L^{2}}(\mathrm{E})}$. Let $\left(u_{n}\right)_{n \in \mathbb{N}} \in \mathcal{C}^{\infty}(\mathrm{E})^{\mathbb{N}}$ such that $u_{n} \rightarrow u$ and $\mathrm{P}_{h} u_{n} \rightarrow \mathrm{P}_{h} u$ in $L^{2}(\mathrm{E})$ when $n \rightarrow+\infty$. Notice that $\pi_{\mathrm{G}} w \in \mathcal{C}^{\infty}(\mathrm{E})$ for all $w \in L^{2}(\mathrm{E})$, because $\pi_{\mathrm{G}}$ is the orthogonal projection on a finite number of smooth functions, namely $\left(\Phi_{j, h} \mathbf{1}_{\mathrm{V}}\right)_{j=1, \ldots, \mathrm{m}_{0}}$. Therefore, $\left(1-\pi_{\mathrm{G}}\right) u_{n} \in \mathcal{C}^{\infty}(\mathrm{E}) \cap \mathrm{G}^{\perp_{L^{2}(\mathrm{E})}}$ and thus, for all $n$, it holds:

$$
\left\|\left(\mathrm{P}_{h}-z\right)\left(1-\pi_{\mathrm{G}}\right) u_{n}\right\|_{L^{2}(\mathrm{E})} \geq \frac{c_{1}}{2} h^{2}\left\|\left(1-\pi_{\mathrm{G}}\right) u_{n}\right\|_{L^{2}(\mathrm{E})}^{2} .
$$

Moreover, $\pi_{\mathrm{G}} u_{n} \rightarrow \pi_{\mathrm{G}} u$ in $L^{2}(\mathrm{E})$ when $n \rightarrow+\infty$ and $\mathrm{P}_{h} \pi_{\mathrm{G}} u_{n} \rightarrow \mathrm{P}_{h} \pi_{\mathrm{G}} u$ in $L^{2}(\mathrm{E})$ when $n \rightarrow+\infty$, because $\mathrm{P}_{h} \pi_{\mathrm{G}}$ is a finite rank operator. Thus passing to the limit in $(28)$, and since $\left(1-\pi_{\mathrm{G}}\right) u=u$, it holds:

$$
\left\|\left(\mathrm{P}_{h}-z\right) u\right\|_{L^{2}(\mathrm{E})} \geq \frac{c_{1}}{2} h^{2}\left\|\left(1-\pi_{\mathrm{G}}\right) u\right\|_{L^{2}(\mathrm{E})}^{2} .
$$

The same analysis leads to the same estimate for $\mathrm{P}_{h}^{*}$. This concludes the proof of Proposition 12 ,

We now proceed to the proof of Theorems 1 and 2 .

\section{Proofs of Theorems 1 and 2}

Let us recall that

$$
\mathrm{P}_{h} \in\left\{\mathrm{P}_{h}^{\mathrm{BPS}}, \mathrm{P}_{h}^{Z Z}\right\}
$$

\subsection{Proof of Theorem 1}

In this section we prove Theorem 1. To this end, we prove the following result.

Proposition 13. Assume that (Morse) holds. Let $c_{1}>0$ be as in (23) and $c_{2}<c_{1} / 2$. Then,

1. There exists $K>0$ and $h_{0}>0$ such that for all $z \in \mathbb{C}$ such that $|z| \geq c_{2} h^{2}$ and $\operatorname{Re} z \leq$ $\frac{c_{1}}{2} h^{2}$, and for all $h \in\left(0, h_{0}\right)$, it holds,

$$
\left\|\left(\mathrm{P}_{h}-z\right)^{-1}\right\| \leq \frac{K}{h^{2}} .
$$

Moreover, there exists $\delta>0$, such that for $h$ small enough $\sigma\left(\mathrm{P}_{h}\right) \cap\left\{\operatorname{Re} \mathrm{z} \leq \frac{c_{1}}{2} h^{2}\right\} \subset\{|\mathrm{z}| \leq$ $\left.e^{-\frac{\delta}{h}}\right\}$. The same holds for $\mathrm{P}_{h}^{*}$ (with different constants). For $\eta \in\left(c_{2}, c_{1} / 2\right)$, the spectral Riesz projection

$$
\pi_{\eta h^{2}}\left(\mathrm{P}_{h}\right)=-\frac{1}{2 i \pi} \int_{|z|=\eta h^{2}}\left(\mathrm{P}_{h}-z\right)^{-1} d z
$$

is thus well defined for $h$ small enough.

2. There exists $\eta_{1}>0$ such that for all $\eta \in\left(0, \eta_{1}\right)$ it holds for all $h$ small enough,

$$
\operatorname{dim} \operatorname{Ran} \pi_{\eta h^{2}}\left(\mathrm{P}_{h}\right)=\mathrm{m}_{0} .
$$

The same holds for $\pi_{\eta h^{2}}\left(\mathrm{P}_{h}^{*}\right)=\pi_{\eta h^{2}}\left(\mathrm{P}_{h}\right)^{*}$. 
Proof. Assume that (Morse) holds. The proof is inspired by [30, Section 2.2]. The main idea is to build a Grushin problem. We refer to [40] for a review on this topic. Let us define the operator

$$
\hat{\mathrm{P}}_{h}=\left(1-\pi_{\mathrm{G}}\right) \mathrm{P}_{h}\left(1-\pi_{\mathrm{G}}\right): D\left(\mathrm{P}_{h}\right) \cap \mathrm{G}^{\perp^{2}(\mathrm{E})} \subset \mathrm{G}^{\perp} L^{2}(\mathrm{E}) \rightarrow \mathrm{G}^{\perp} L^{2}(\mathrm{E}),
$$

which is well defined because $\pi_{\mathrm{G}} L^{2}(\mathrm{E}) \subset \mathcal{C}^{\infty}(\mathrm{E}) \subset D\left(\mathrm{P}_{h}\right)$. We equip $\mathrm{G}^{\perp^{2}(\mathrm{E})}$ with the Hermitian inner product of $L^{2}(\mathrm{E})$. This is a Hilbert space. Notice that $\hat{\mathrm{P}}_{h}$ is a closed (it is the sum of $\mathrm{P}_{h}$ and the finite rank operator $-\pi_{\mathrm{G}} \mathrm{P}_{h}-\mathrm{P}_{h} \pi_{\mathrm{G}}+\pi_{\mathrm{G}} \mathrm{P}_{h} \pi_{\mathrm{G}}$ ) and densely defined on $\mathrm{G}^{\perp_{L^{2}}(\mathrm{E})}$ (indeed, for $u \in \mathrm{G}^{\perp_{L^{2}}(\mathrm{E})}$, there exists $\left(u_{n}\right)_{n \geq 0} \in \mathcal{C}^{\infty}(\mathrm{E})$ such that $u_{n} \rightarrow u$ in $L^{2}(\mathrm{E})$ and thus $\mathrm{G}^{\perp^{2}(\mathrm{E})} \cap \mathcal{C}^{\infty}(\mathrm{E}) \ni\left(1-\pi_{\mathrm{G}}\right) u_{n} \rightarrow\left(1-\pi_{\mathrm{G}}\right) u=u$ in $\left.L^{2}(\mathrm{E})\right)$.

Step 1. Let us prove that the operator $\hat{\mathrm{P}}_{h}-z$ is invertible for $\operatorname{Re} z \leq \frac{c_{1}}{2} h^{2}$ and $h$ small enough (up to choosing $c_{1}$ smaller, where $c_{1}$ is as in (23)).

From (23) and the Pythagorean Theorem, if $\operatorname{Re} z \leq \frac{c_{1}}{2} h^{2}$, there exists $h_{0}>0$ such that for all $h \in\left(0, h_{0}\right)$ and for all $u \in D\left(\mathrm{P}_{h}\right) \cap \mathrm{G}^{{ }{ }^{2}(\mathrm{E})}$ :

$$
\left\|\left(\hat{\mathrm{P}}_{h}-z\right) u\right\|_{L^{2}(\mathrm{E})} \geq \frac{c_{1}}{2} h^{2}\|u\|_{L^{2}(\mathrm{E})} .
$$

This proves that $\left(\hat{\mathrm{P}}_{h}-z\right)$ is injective with closed range. Let us now prove that its range is dense. To this end, we need to identify the adjoint $\hat{\mathrm{P}}_{h}^{\dagger}$ of $\hat{\mathrm{P}}_{h}$ to make use of the relation

$$
\operatorname{Ran}\left(\hat{\mathrm{P}}_{h}-z\right)=\operatorname{Ker}\left(\hat{\mathrm{P}}_{h}^{\dagger}-z\right)^{\perp}
$$

which holds because $\hat{P}_{h}$ is a closed accretive operator. Recall that $\hat{P}_{h}$ is the sum of $\mathrm{P}_{h}$ and the bounded operator $-\pi_{\mathrm{G}} \mathrm{P}_{h}-\mathrm{P}_{h} \pi_{\mathrm{G}}+\pi_{\mathrm{G}} \mathrm{P}_{h} \pi_{\mathrm{G}}$. Therefore, the adjoint of $\left(\hat{\mathrm{P}}_{h}, D\left(\mathrm{P}_{h}^{*}\right) \cap \mathrm{G}^{\perp} L^{2}(\mathrm{E})\right)$ on $\mathrm{G}^{\perp} L^{2}(\mathrm{E})$ is the operator

$$
\hat{\mathrm{P}}_{h}^{*}=\left(1-\pi_{\mathrm{G}}\right) \mathrm{P}_{h}^{*}\left(1-\pi_{\mathrm{G}}\right)
$$

with domain $D\left(\mathrm{P}_{h}\right) \cap \mathrm{G}^{\perp_{L^{2}}(\mathrm{E})}$ (recall that $D\left(\mathrm{P}_{h}^{*}\right)=D\left(\mathrm{P}_{h}\right)$, see Proposition 6).

To prove that the range of $\hat{\mathrm{P}}_{h}-z$ is dense, it is thus sufficient to prove that $\hat{\mathrm{P}}_{h}^{*}-z$ is injective. But this follows from the fact that since $\mathrm{P}_{h}^{*}\left(1-\pi_{\mathrm{G}}\right)=\hat{\mathrm{P}}_{h}^{*}$ also satisfies a resolvent estimate (23) on

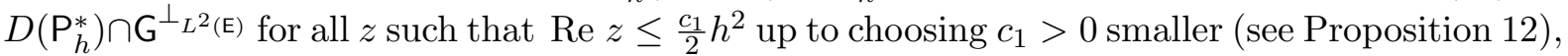
$\left(1-\pi_{\mathrm{G}}\right) \mathrm{P}_{h}^{*}\left(1-\pi_{\mathrm{G}}\right)-z$ is injective. In conclusion, $\hat{\mathrm{P}}_{h}-z$ is invertible for all $z \in \mathbb{C}$ such that $\operatorname{Re} z \leq \frac{c_{1}}{2} h^{2}$ and it holds:

$$
\left\|\left(\hat{\mathrm{P}}_{h}-z\right)^{-1}\right\| \leq \frac{2}{c_{1} h^{2}}
$$

Here $\left\|\left(\hat{\mathrm{P}}_{h}-z\right)^{-1}\right\|$ denotes the norm of $\left(\hat{\mathrm{P}}_{h}-z\right)^{-1} \in \mathcal{L}\left(\mathrm{G}^{\perp} L^{2(\mathrm{E})}\right)$.

Step 2. Grushin problem.

We define the operators (see (11)):

$$
\mathrm{R}_{-}: \mathbb{C}^{\mathrm{m}_{0}} \rightarrow L^{2}(\mathrm{E}), \quad\left(\mu_{k}\right)_{j=1, \ldots, \mathrm{m}_{0}} \mapsto \sum_{j=1}^{\mathrm{m}_{0}} \mu_{j} \Phi_{j, h} \mathbf{1}_{\mathrm{V}},
$$

and

$$
\mathrm{R}_{+}: L^{2}(\mathrm{E}) \rightarrow \mathbb{C}^{\mathrm{m}_{0}}, u \mapsto\left(\left\langle\Phi_{j, h} \mathbf{1}_{\mathrm{V}}, u\right\rangle_{L^{2}(\mathrm{E})}\right)_{j=1, \ldots, \mathrm{m}_{0}} .
$$

We equip $\mathbb{C}^{\mathrm{m}_{0}}$ with the $\ell_{2}$ norm. Notice that

$$
\mathrm{R}_{+} \mathrm{R}_{-}=\mathrm{I}_{\mathbb{C}^{\mathrm{m}_{0}}} \text { and } \mathrm{R}_{-} \mathrm{R}_{+}=\pi_{\mathrm{G}}
$$


In addition, one has:

$$
\left\|\mathrm{R}_{+}\right\| \leq \sqrt{\mathrm{m}_{0}},\left\|\mathrm{R}_{-}\right\| \leq \sqrt{\mathrm{m}_{0}},\left\|\mathrm{R}_{+} \mathrm{P}_{h}\right\| \leq e^{-\frac{c}{h}} \text { and }\left\|\mathrm{P}_{h} \mathrm{R}_{-}\right\| \leq e^{-\frac{c}{h}}
$$

where the two previous inequalities follow from $(12)$ and hold for $h$ small enough $(c>0$ is independent of $h$ ). For $z \in \mathbb{C}$, let us denote by $\mathcal{P}_{h}(z)$ the linear operator defined by

$$
\mathcal{P}_{h}(z):\left(u, u_{-}\right) \in D\left(\mathrm{P}_{h}\right) \times \mathbb{C}^{\mathrm{m}_{0}} \mapsto\left(\begin{array}{c}
\left(\mathrm{P}_{h}-z\right) u+\mathrm{R}_{-} u_{-} \\
\mathrm{R}_{+} u
\end{array}\right) .
$$

Step 2a. The Grushin problem is well posed.

Let us prove that the previous Grushin problem is well posed, i.e. let us prove that $\mathcal{P}_{h}(z)$ : $D\left(\mathrm{P}_{h}\right) \times \mathbb{C}^{\mathrm{m}_{0}} \rightarrow L^{2}(\mathrm{E}) \times \mathbb{C}^{\mathrm{m}_{0}}$ is invertible for Re $z \leq \frac{c_{1}}{2} h^{2}$ and $h$ small enough. Let $z \in \mathbb{C}$ such that $\operatorname{Re} z \leq \frac{c_{1}}{2} h^{2}$ and $(f, y) \in L^{2}(\mathrm{E}, \mu) \times \mathbb{C}^{\mathrm{m}_{0}}$. Assume that $\left(u, u_{-}\right) \in D\left(\mathrm{P}_{h}\right) \times \mathbb{C}^{\mathrm{m}_{0}}$ satisfies

$$
\mathcal{P}_{h}(z)\left(u, u_{-}\right)=(f, g) .
$$

By definition, the previous equality means that

$$
\left(\mathrm{P}_{h}-z\right) u+\mathrm{R}_{-} u_{-}=f \text { and } \mathrm{R}_{+} u=y .
$$

Applying $\mathrm{R}_{+}$to the first equation and $\mathrm{R}_{-}$to the second leads to $\mathrm{R}_{+}\left(\mathrm{P}_{h}-z\right) u+u_{-}=\mathrm{R}_{+} f$ and $\pi_{\mathrm{G}} u=\mathrm{R}_{-} y$ (see (32)). Write $u=\pi_{\mathrm{G}} u+\left(1-\pi_{\mathrm{G}}\right) u=\mathrm{R}_{-} y+\left(1-\pi_{\mathrm{G}}\right) u$. It thus remains to find $\left(1-\pi_{\mathrm{G}}\right) u$. One has $\left(\mathrm{P}_{h}-z\right)\left(1-\pi_{\mathrm{G}}\right) u+\left(\mathrm{P}_{h}-z\right) \mathrm{R}_{-} y+\mathrm{R}_{-} u_{-}=f$, and applying $\left(1-\pi_{\mathrm{G}}\right)$ leads to

$$
\hat{\mathrm{P}}_{h}\left(1-\pi_{\mathrm{G}}\right) u=\left(1-\pi_{\mathrm{G}}\right) f-\left(1-\pi_{\mathrm{G}}\right) \mathrm{P}_{h} \mathrm{R}_{-} y,
$$

where we have used that $\left(1-\pi_{\mathrm{G}}\right) \mathrm{R}_{-}=0$. Thus, it holds:

$$
u=\mathrm{R}_{-} y+v \text { and } u_{-}=\mathrm{R}_{+} f-\mathrm{R}_{+}\left(\mathrm{P}_{h}-z\right)\left[\mathrm{R}_{-} y+v\right],
$$

where

$$
v=\left(\hat{\mathrm{P}}_{h}-z\right)^{-1}\left(1-\pi_{\mathrm{G}}\right) f-\left(\hat{\mathrm{P}}_{h}-z\right)^{-1}\left(1-\pi_{\mathrm{G}}\right) \mathrm{P}_{h} \mathrm{R}_{-} y .
$$

Let us now choose $z$ such that Re $z \leq \frac{c_{1}}{2} h^{2}$ and $h$ small enough. Take $(f, y) \in L^{2}(\mathrm{E}, \mu) \times \mathbb{C}^{\mathrm{m}_{0}}$ and

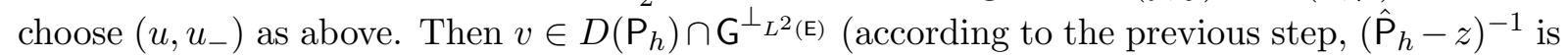
well defined on $\mathrm{G}^{\perp_{L^{2}}(\mathrm{E})}$ and its range is $\left.D\left(\mathrm{P}_{h}\right) \cap \mathrm{G}^{\perp^{2}(\mathrm{E})}\right)$. Therefore $u \in D\left(\mathrm{P}_{h}\right)$. Since $\mathrm{R}_{+} v=0$, it holds $\mathrm{R}_{+} u=y$. Moreover, using (32), it is straightforward to check that $\left(\mathrm{P}_{h}-z\right) u+\mathrm{R}_{-} u_{-}=f$. This proves that the previous Grushin problem is well posed. Write the inverse of $\mathcal{P}_{h}(z)$ as, for $\operatorname{Re} z \leq \frac{c_{1}}{2} h^{2}$ and $h$ small enough,

$$
(f, y) \in L^{2}(\mathrm{E}) \times \mathbb{C}^{\mathrm{m}_{0}} \mapsto\left(\begin{array}{cc}
\mathcal{E}(z) & \mathcal{E}_{+}(z) \\
\mathcal{E}_{-}(z) & \mathcal{E}_{-+}(z)
\end{array}\right)\left(\begin{array}{l}
f \\
y
\end{array}\right),
$$

where the operators $\mathcal{E}(z), \mathcal{E}_{+}(z), \mathcal{E}_{-}(z)$, and $\mathcal{E}_{-+}(z)$ equal:

1. $\mathcal{E}(z)=\left(\hat{\mathrm{P}}_{h}-z\right)^{-1}\left(1-\pi_{\mathrm{G}}\right)$ is holomorphic for Re $z \leq \frac{c_{1}}{2} h^{2}$. Using (31), it holds for $\operatorname{Re} z \leq \frac{c_{1}}{2} h^{2}$

$$
\|\mathcal{E}(z)\| \leq \frac{2}{c_{1} h^{2}}
$$

2. $\mathcal{E}_{-+}(z)=-\mathrm{R}_{+}\left(\mathrm{P}_{h}-z\right) \mathrm{R}_{-}+\mathrm{R}_{+}\left(\mathrm{P}_{h}-z\right)\left(\hat{\mathrm{P}}_{h}-z\right)^{-1}\left(1-\pi_{\mathrm{G}}\right) \mathrm{P}_{h} \mathrm{R}_{-}$. 
3. $\mathcal{E}_{+}(z)=\mathrm{R}_{-}-\left(\hat{\mathrm{P}}_{h}-z\right)^{-1}\left(1-\pi_{\mathrm{G}}\right) \mathrm{P}_{h} \mathrm{R}_{-}$.

4. $\mathcal{E}_{-}(z)=\mathrm{R}_{+}-\mathrm{R}_{+} \mathrm{P}_{h}\left(\hat{\mathrm{P}}_{h}-z\right)^{-1}\left(1-\pi_{\mathrm{G}}\right)$ (notice that we have used here that $u_{-}=\mathrm{R}_{+} f-$ $\mathrm{R}_{+}\left(\mathrm{P}_{h}-z\right) \mathrm{R}_{-} y-\mathrm{R}_{+} \mathrm{P}_{h} v$ because $\left.\mathrm{R}_{+} v=\mathrm{R}_{+}\left(1-\pi_{\mathrm{G}}\right) v=0\right)$.

Step 2b. End of the proof of Proposition 13 .

In the following $\operatorname{Re} z \leq \frac{c_{1}}{2} h^{2}$ and $h$ is small enough. Let us recall that $\mathrm{P}_{h}-z$ is invertible if and only if $\mathcal{E}_{-+}(z)$ is invertible (see $[40]$ ) and in this case,

$$
\left(\mathrm{P}_{h}-z\right)^{-1}=\mathcal{E}(z)-\mathcal{E}_{+}(z) \mathcal{E}_{-+}(z)^{-1} \mathcal{E}_{-}(z) .
$$

From (31) and (33), there exists $c>0$ such that for $h$ small enough:

$$
\left\|\mathcal{E}_{-}(z)-\mathrm{R}_{+}\right\| \leq e^{-\frac{c}{h}} \text { and }\left\|\mathcal{E}_{+}(z)-\mathrm{R}_{-}\right\| \leq e^{-\frac{c}{h}} .
$$

Let us now estimate $\mathcal{E}_{-+}(z)$. First, one has using (33),

$$
-\mathrm{R}_{+}\left(\mathrm{P}_{h}-z\right) \mathrm{R}_{-}=\left.z\right|_{\mathbb{C}^{\mathrm{m}_{0}}}+O\left(e^{-\frac{c}{h}}\right) .
$$

Secondly, $\mathrm{R}_{+}\left(\mathrm{P}_{h}-z\right)\left(\hat{\mathrm{P}}_{h}-z\right)^{-1}\left(1-\pi_{\mathrm{G}}\right) \mathrm{P}_{h} \mathrm{R}_{-}=\mathrm{R}_{+} \mathrm{P}_{h}\left(\hat{\mathrm{P}}_{h}-z\right)^{-1}\left(1-\pi_{\mathrm{G}}\right) \mathrm{P}_{h} \mathrm{R}_{-}$because $\mathrm{R}_{+}=0$ on $\mathrm{G}^{{ }^{2}{ }^{2}(\mathrm{E})}$. Thus, using (31) and (33),

$$
\left\|\mathrm{R}_{+}\left(\mathrm{P}_{h}-z\right)\left(\hat{\mathrm{P}}_{h}-z\right)^{-1}\left(1-\pi_{\mathrm{G}}\right) \mathrm{P}_{h} \mathrm{R}_{-}\right\| \leq e^{-\frac{c}{h}} .
$$

Thus, $\mathcal{E}_{-+}(z)=z \mathbf{l}_{\mathbb{C}^{m_{0}}}+O\left(e^{-\frac{c}{h}}\right)$. Let $c_{2} \in\left(0, c_{1} / 2\right)$. Then, the operator $\mathcal{E}_{-+}(z)$ is invertible for $z \in \mathbb{C}$ such that $|z| \geq c_{2} h^{2}$ and Re $z \leq \frac{c_{1}}{2} h^{2}$, and for $h$ small enough. In this case, one has $\mathcal{E}_{-+}(z)^{-1}=z^{-1}\left(1+O\left(e^{-\frac{c}{h}}\right)\right)$. Hence, from $(35)$, since $\mathrm{R}_{+} \mathrm{R}_{-}=\pi_{\mathrm{G}},\left\|\pi_{\mathrm{G}}\right\|=1,\left\|\mathrm{R}_{+}\right\| \leq \sqrt{\mathrm{m}_{0}}$, and $\left\|\mathrm{R}_{-}\right\| \leq \sqrt{\mathrm{m}_{0}}$, the previous estimates on $\mathcal{E}_{+}(z), \mathcal{E}_{-}(z)$, and $\mathcal{E}_{-+}(z)$ imply that there exists $c>0$ such that:

$$
\left(\mathrm{P}_{h}-z\right)^{-1}=\mathcal{E}(z)-z^{-1}\left(\pi_{\mathrm{G}}+O\left(e^{-\frac{c}{h}}\right)\right),
$$

for all $h$ small enough. From (34), if $|z| \geq c_{2} h^{2}$ and Re $z \leq \frac{c_{1}}{2} h^{2}$, one has for $h$ small enough:

$$
\left\|\left(\mathrm{P}_{h}-z\right)^{-1}\right\| \leq \frac{2}{c_{1} h^{2}}+\frac{\left(1+O\left(e^{-\frac{c}{h}}\right)\right)}{|z|} \leq \frac{K}{h^{2}},
$$

where $K>0$ is a constant independent of $z$ and $h$. The same estimates also holds for $\mathrm{P}_{h}^{*}$ with different constants $c_{1}$ and $c_{2}$. Notice also that there exists $\delta>0$ (not too large) such that if $|z| \geq e^{-\frac{\delta}{h}}$ and $\operatorname{Re} z \leq \frac{c_{1}}{2} h^{2}$, for $h$ small enough, $\mathcal{E}_{-+}(z)$ is invertible and thus so is $\mathrm{P}_{h}-z$ (see (35)). Therefore, for $h$ small enough:

$$
\sigma\left(\mathrm{P}_{h}\right) \cap\left\{\operatorname{Re} \mathrm{z} \leq \frac{c_{1}}{2} h^{2}\right\} \subset\left\{|\mathrm{z}| \leq e^{-\frac{\delta}{h}}\right\} .
$$

This concludes the proof of item 1 in Proposition 13 . Moreover, from (29) and (36), one has

$$
\pi_{\eta h^{2}}\left(\mathrm{P}_{h}\right)=\pi_{\mathrm{G}}+O\left(e^{-\frac{c}{h}}\right) .
$$

This concludes the proof of Proposition 13 because $\pi_{\mathrm{G}}$ is a $\mathrm{m}_{0}$ dimensional projection.

Let us finally give an estimate which will be used in the proof of Theorem 2 below. Let $z$ be such that Re $z \leq \frac{c_{1}}{2} h^{2}$. For all $\left(u, u_{-}\right) \in D\left(\mathrm{P}_{h}\right) \times \mathbb{C}^{\mathrm{m}_{0}}$, consider $(f, y) \in L^{2}(\mathrm{E}) \times \mathbb{C}^{\mathrm{m}_{0}}$ such that $\mathcal{P}_{h}(z)\left(u, u_{-}\right)=(f, y)$. Then, $u=\mathcal{E}(z) f+\mathcal{E}_{+}(z) y$. The previous estimates (see (29)) imply that for all $z$ such that $\operatorname{Re} z \leq \frac{c_{1}}{2} h^{2}$, for all $h$ small enough and all $u \in D\left(\mathrm{P}_{h}\right)$,

$$
\|u\|_{L^{2}(\mathrm{E})} \leq \frac{2}{c_{1} h^{2}}\|f\|_{L^{2}(\mathrm{E})}+\left(\sqrt{\mathrm{m}_{0}}+e^{-\frac{c}{h}}\right)\|y\|_{\ell_{2}},
$$

for some $c>0$ independent of $z, h$ and $u$. 
To conclude the proof of Theorem 1 , in view of Proposition 13, it remains to show that in a disk of radius smaller than $\eta h^{2}$, the spectrum of $\mathrm{P}_{h}$ is made of real eigenvalues. To this end we will use the fact that the operator $\mathrm{P}_{h}$ admits a PT-symmetry property (for Parity Time-symmetry). We refer to 25,39] for more details and references on this topic.

Let us define the operator,

$$
\mathrm{X}: u \in \mathrm{L}^{2}(\mathrm{E}) \mapsto \mathrm{X} u(x, v)=u(x,-v) \in \mathrm{L}^{2}(\mathrm{E}) .
$$

The operator $\mathrm{X}$ is unitary, self-adjoint, and $\mathrm{X}^{-1}=\mathrm{X}$ in $\mathrm{L}^{2}(\mathrm{E})$. Moreover, one has (see Proposition 6),

$$
\mathrm{P}_{h}^{*}=\mathrm{X}^{-1} \mathrm{P}_{h} \mathrm{X}
$$

Let us finally define the bilinear form,

$$
u, v \in \mathrm{L}^{2}(\mathrm{E}) \mapsto\langle u, v\rangle_{\mathbf{X}}=\langle\mathrm{X} u, v\rangle_{\mathrm{L}^{2}(\mathrm{E})} .
$$

We have the following result.

Lemma 14. Assume that (Morse) hold. Then $u, v \in L^{2}(\mathrm{E}) \mapsto\langle u, v\rangle_{\mathrm{X}}$ restricted to the Range of $\pi_{\eta h^{2}}\left(\mathrm{P}_{h}\right)$ (see (29)) is an Hermitian inner product uniformly in $h$ small enough.

Proof. We just have to check that $u, v \in \operatorname{Ran}\left(\pi_{\eta h^{2}}\left(\mathrm{P}_{h}\right)\right) \mapsto\langle u, v\rangle_{\mathbf{X}}$ is positive-definite uniformly in $h$ small enough. From (12), there exists $c>0$ such that for $h$ small enough, it holds for all $j \in\left\{1, \ldots, \mathrm{m}_{0}\right\}$ :

$$
\left(\mathrm{P}_{h}-z\right) \Phi_{j, h} 1_{\vee}=-z \Phi_{j, h} 1_{\vee}+O\left(e^{-\frac{c}{h}}\right) .
$$

Let $z \in \mathbb{C}$ such that $|z| \geq c_{2} h^{2}$ and Re $z \leq \frac{c_{1}}{2} h^{2}$. Using item 1 in Proposition 13 , one deduces that for $h$ small enough:

$$
\left(\mathrm{P}_{h}-z\right)^{-1} \Phi_{j, h} 1_{\vee}=-z^{-1}\left[\Phi_{j, h} 1_{\vee}+O\left(e^{-\frac{c}{h}}\right)\right] .
$$

Thus 290 implies that

$$
\pi_{\eta h^{2}}\left(\mathrm{P}_{h}\right)\left(\Phi_{j, h} 1_{\mathrm{V}}\right)=\Phi_{j, h} 1_{\vee}+O\left(e^{-\frac{c}{h}}\right)\left(\text { and the same holds for } \pi_{\eta h^{2}}\left(\mathrm{P}_{h}^{*}\right)=\pi_{\eta h^{2}}\left(\mathrm{P}_{h}\right)^{*}\right) .
$$

Thus, for $h$ small enough, one has:

$$
\left\langle\pi_{\eta h^{2}}\left(\mathrm{P}_{h}\right)\left(\Phi_{j, h} \mathbf{1}_{\vee}\right), \pi_{\eta h^{2}}\left(\mathrm{P}_{h}\right)\left(\Phi_{i, h} \mathbf{1}_{\vee}\right)\right\rangle_{L^{2}(\mathrm{E})}=\delta_{i, j}+O\left(e^{-\frac{c}{h}}\right) .
$$

Since $\pi_{\eta h^{2}}\left(\mathrm{P}_{h}\right)$ is of rank $\mathrm{m}_{0}$ for $h$ small enough, one deduces that for $h$ small enough,

$$
\left\{\pi_{\eta h^{2}}\left(\mathrm{P}_{h}\right)\left(\Phi_{1, h} \mathbf{1}_{\mathbf{V}}\right), \ldots, \pi_{\eta h^{2}}\left(\mathrm{P}_{h}\right)\left(\Phi_{\mathrm{m}_{0}, h} \mathbf{1}_{\mathbf{V}}\right)\right\} \text { is a basis of } \operatorname{Ran} \pi_{\eta h^{2}}\left(\mathrm{P}_{h}\right) .
$$

Let $j \in\left\{1, \ldots, \mathbf{m}_{0}\right\}$. Since $\mathbf{X}\left(\Phi_{j, h} \mathbf{1}_{\mathbf{V}}\right)=\Phi_{j, h} \mathbf{1}_{\mathbf{V}}$, from 40, , one has:

$$
\left\langle\pi_{\eta h^{2}}\left(\mathrm{P}_{h}\right)\left(\Phi_{j, h} \mathbf{1}_{\mathbf{V}}\right), \pi_{\eta h^{2}}\left(\mathrm{P}_{h}\right)\left(\Phi_{i, h} \mathbf{1}_{\mathbf{V}}\right)\right\rangle_{\mathbf{X}}=\delta_{i, j}+O\left(e^{-\frac{c}{h}}\right) .
$$

Therefore, for $h$ small enough and for all $w \in \operatorname{Ran}\left(\pi_{\eta h^{2}}\left(\mathrm{P}_{h}\right)\right)$, writing

$$
w=\sum_{j=1}^{\mathrm{m}_{0}} w_{j, h} \pi_{\eta h^{2}}\left(\mathrm{P}_{h}\right) \Phi_{j, h} \mathbf{1}_{\mathbf{v}}
$$


where $w_{j, h} \in \mathbb{R}$, one deduces that for $h$ small enough

$$
\langle w, w\rangle_{\mathbf{X}}=\left(1+O\left(e^{-\frac{c}{h}}\right)\right) \sum_{j=1}^{\mathrm{m}_{0}}\left|w_{j, h}\right|^{2},
$$

where $c>0$ is independent of $h$ and $w$. Therefore, uniformly in $h$ small enough, $\langle\cdot, \cdot\rangle_{\mathbf{X}}$ is an Hermitian inner product when restricted to $\operatorname{Ran}\left(\pi_{\eta h^{2}}\left(\mathrm{P}_{h}\right)\right)$. This concludes the proof of the lemma. Notice that the same holds true for $\langle w, w\rangle_{L^{2}(\mathrm{E})}$ and thus for $h$ small enough, the Hermitian inner products

$$
w \mapsto\langle w, w\rangle_{\mathbf{X}} \text { and } w \mapsto\langle w, w\rangle_{L^{2}(\mathrm{E})} \text { are equivalent on } \operatorname{Ran}\left(\pi_{\eta h^{2}}\left(\mathrm{P}_{h}\right)\right),
$$

where the equivalent constants are of order $1+O\left(e^{-\frac{c}{h}}\right)$.

Let us now end the proof of Theorem 1. According to item 2 in Proposition 13 and from the Riesz decomposition theorem of the spectrum (see for instance [41, Theorem 3.14.10] or [29, Theorem 6.17]), the spectrum of $\mathrm{P}_{h}$ lying inside the disk of radius $\eta h^{2}$ is the spectrum of the square matrix $\mathrm{M}_{h}$ of size $\mathrm{m}_{0}$ of

$$
\mathrm{P}_{h}: \operatorname{Ran}\left(\pi_{\eta h^{2}}\left(\mathrm{P}_{h}\right)\right) \rightarrow \operatorname{Ran}\left(\pi_{\eta h^{2}}\left(\mathrm{P}_{h}\right)\right),
$$

computed for instance in an orthonormal basis of $\operatorname{Ran}\left(\pi_{\eta h^{2}}\left(\mathrm{P}_{h}\right)\right)$ for the Hermitian inner product $\langle\cdot, \cdot\rangle_{\mathrm{X}}$ (see Lemma 14). This implies that the spectrum of $\mathrm{P}_{h}$ lying inside the disk of radius $\eta h^{2}$ is composed of a finite number of eigenvalues of finite algebraic multiplicities. Furthermore, from (38), $\mathrm{M}_{h}$ is symmetric. Thus, these eigenvalues are real and their algebraic multiplicity equals their geometric multiplicity. From item 1 in Proposition 13 , these $m_{0}$ eigenvalues are actually exponentially small in the limit $h \rightarrow 0$. The same arguments also apply to $\mathrm{P}_{h}^{*}$. This concludes the proof of Theorem 1 (as already mentioned, the statement concerning the eigenvalue 0 in Theorem 1 is true for all $h>0$, see Proposition 16 below).

\subsection{Proof of Theorem 2}

In this section we prove Theorem 2. Let us assume that (Morse) holds. Let us denote by $\lambda_{1}, \ldots, \lambda_{\mathrm{m}_{0}}$ the $\mathrm{m}_{0}$ smallest eigenvalues of $\mathrm{P}_{h}$, which are real and exponentially small when $h \rightarrow 0$ according to Theorem 1. Let $\Pi_{\lambda_{j}}\left(\mathrm{P}_{h}\right), j=1, \ldots, \mathrm{m}_{0}$, be the spectral projection associated with $\lambda_{j}$ for $\mathrm{P}_{h}$.

Remark 15. In [35], in a one-dimensional case, it is shown that the spectrum of $\mathrm{P}_{h}$ contains a sequence $\left(\lambda_{n}\right)_{n \geq 0}$ such that $\operatorname{Re} \lambda_{n}$ is bounded and $\left|\operatorname{Im} \lambda_{n}\right| \rightarrow \infty$ as $n \rightarrow+\infty$. This suggests that the contour deformation procedure made in [21,26] on the semigroup $\left(e^{-t \mathrm{P}_{h}}\right)_{t \geq 0}$ might not be successful for $\mathrm{P}_{h}$. Thus, we rather use a resolvent estimate on $\mathrm{P}_{h}\left(1-\pi_{\eta h^{2}}\left(\mathrm{P}_{h}\right)\right)$ and a quantitative version of the Gearhart-Prüss Theorem, see [19, Section 13] and [23].

Let us recall that from Proposition 12 , for all all $c_{1} \in\left(0, c_{0}\right)$, if $\operatorname{Re} z \leq \frac{c_{1}}{2} h^{2}$, there exists $h_{0}>0$ such that for all $h \in\left(0, h_{0}\right)$ and for all $u \in D\left(\mathrm{P}_{h}\right) \cap \mathrm{G}^{\perp} L^{2}(\mathrm{E})$ :

$$
\left\|\left(\mathrm{P}_{h}-z\right) u\right\|_{L^{2}(\mathrm{E})} \geq \frac{c_{1}}{2} h^{2}\|u\|_{L^{2}(\mathrm{E})} .
$$

Let us show that this implies a similar resolvent estimate for $\mathrm{P}_{h}\left(1-\pi_{\eta h^{2}}\left(\mathrm{P}_{h}\right)\right)$. Let $w \in(1-$ $\left.\pi_{\eta h^{2}}\left(\mathrm{P}_{h}\right)\right) D\left(\mathrm{P}_{h}\right)$. Then, one has, using (39):

$\left\langle w, \Phi_{j, h} \mathbf{1}_{\mathbf{V}}\right\rangle_{L^{2}(\mathrm{E})}=\left\langle\left(1-\pi_{\eta h^{2}}\left(\mathrm{P}_{h}\right)\right) w, \Phi_{j, h} \mathbf{1}_{\mathbf{V}}\right\rangle_{L^{2}(\mathrm{E})}=\left\langle w,\left(1-\pi_{\eta h^{2}}\left(\mathrm{P}_{h}\right)^{*}\right) \Phi_{j, h} \mathbf{1}_{\mathbf{V}}\right\rangle_{L^{2}(\mathrm{E})} \leq e^{-\frac{c}{h}}\|w\|_{L^{2}(\mathrm{E})}$. 
This implies that $\left\|\mathrm{R}_{+} w\right\|_{\ell_{2}} \leq e^{-\frac{c}{h}}\|w\|_{L^{2}(\mathrm{E})}$. Let $z$ be such that $\operatorname{Re} z \leq \frac{c_{1}}{2} h^{2}$. Then, setting $u_{-}=0$ and $u=w$ in (37), it holds $y=\mathrm{R}_{+} w$ and $f=\left(\mathrm{P}_{h}-z\right) w$, and then:

$$
\|w\|_{L^{2}(\mathrm{E})} \leq \frac{2}{c_{1} h^{2}}\|f\|_{L^{2}(\mathrm{E})}+\left(\sqrt{\mathrm{m}_{0}}+e^{-\frac{c}{h}}\right) e^{-\frac{c}{h}}\|w\|_{L^{2}(\mathrm{E})},
$$

for some $c>0$ independent of $z, h$ and $w$. This implies that for all $u \in D\left(\mathrm{P}_{h}\right)$, for $h$ small enough,

$$
\frac{1}{2}\left\|\left(1-\pi_{\eta h^{2}}\left(\mathrm{P}_{h}\right)\right) u\right\|_{L^{2}(\mathrm{E})} \leq \frac{2}{c_{1} h^{2}}\left\|\left(\mathrm{P}_{h}-z\right)\left(1-\pi_{\eta h^{2}}\left(\mathrm{P}_{h}\right)\right) u\right\|_{L^{2}(\mathrm{E})} .
$$

Let us denote by $\tilde{\mathrm{P}}_{h}$ the operator $\mathrm{P}_{h}$ with domain $\left(1-\pi_{\eta h^{2}}\left(\mathrm{P}_{h}\right)\right) D\left(\mathrm{P}_{h}\right)$ on the closed subspace $\mathrm{F}=\left(1-\pi_{\eta h^{2}}\left(\mathrm{P}_{h}\right)\right) L^{2}(\mathrm{E})$ of $L^{2}(\mathrm{E})$. Let us recall that, for $h>0, \tilde{\mathrm{P}}_{h}$ is the generator of the strongly continuous contraction semigroup $\left(\left.e^{-t \mathrm{P}_{h}}\right|_{\mathrm{F}}\right)_{t \geq 0}$ (see for instance [41, Theorem 3.14.10]) and is thus $m$-accretive from the Hille-Yosida Theorem. Let us show that $\tilde{\mathrm{P}}_{h}-z$ is invertible for all $z$ such that Re $z \leq \frac{c_{1}}{2} h^{2}$ and for all $h$ small enough. Equation (44) implies that $\tilde{\mathrm{P}}_{h}-z$ is injective with closed range for all $h$ small enough and for all $z$ such that $\operatorname{Re} z \leq \frac{c_{1}}{2} h^{2}$. In particular, it is a semi-Fredholm operator. Since $\tilde{\mathrm{P}}_{h}$ is $m$-accretive for $h>0, \tilde{\mathrm{P}}_{h}-z$ is invertible when $z \in \mathbb{R}_{-}^{*}$ and thus its index is 0 . This implies that, since the index is constant on the connected set $\operatorname{Re} z \leq \frac{c_{1}}{2} h^{2}$ (see [29, Theorem 5.17]), that the index of $\tilde{\mathrm{P}}_{h}-z$ is 0 when $\operatorname{Re} z \leq \frac{c_{1}}{2} h^{2}$. Thus, $\operatorname{Ran}\left(\tilde{\mathrm{P}}_{h}-z\right)=\mathrm{F}$. Consequently, for all $h$ small enough and for all $z$ such that $\operatorname{Re} z \leq \frac{c_{1}}{2} h^{2}$, $\tilde{\mathrm{P}}_{h}-z$ is invertible and one has the resolvent estimate

$$
\left\|\left(\tilde{\mathrm{P}}_{h}-z\right)^{-1}\right\| \leq \frac{4}{c_{1} h^{2}} .
$$

The previous resolvent estimate for $\tilde{\mathrm{P}}_{h}$ implies, applying [23, Proposition 2.1] to $\left.e^{-t \mathrm{P}_{h}}\right|_{\mathrm{F}}$ (see also [19, Proposition 13.31]), that for all $h>0$ small enough, one has for all $u \in L^{2}(\mathrm{E})$ and all $t \geq 0$ :

$$
\left\|e^{-t \mathrm{P}_{h}}\left(1-\pi_{\eta h^{2}}\left(\mathrm{P}_{h}\right)\right) u\right\| \leq\left[1+2 \frac{c_{1} h^{2} / 2}{c_{1} h^{2} / 4}\right] e^{-\frac{c_{1}}{2} h^{2} t}\left(1+\left\|\pi_{\eta h^{2}}\left(\mathrm{P}_{h}\right)\right\|\right)\|u\|_{L^{2}(\mathrm{E})} .
$$

Moreover, we have:

$$
\pi_{\eta h^{2}}\left(\mathrm{P}_{h}\right)=\sum_{j=1}^{\mathrm{m}_{0}} \Pi_{\lambda_{j}}\left(\mathrm{P}_{h}\right) \text { and for all } j \text { and for all } t \geq 0, e^{-t \mathrm{P}_{h}} \Pi_{\lambda_{j}}\left(\mathrm{P}_{h}\right)=e^{-t \lambda_{j}} \Pi_{\lambda_{j}}\left(\mathrm{P}_{h}\right) .
$$

In addition, from (43), for all $j=1, \ldots, \mathrm{m}_{0}$ and $h$ small enough, $\left\|\Pi_{j}\right\| \leq C\left\|\Pi_{\lambda_{j}}\left(\mathrm{P}_{h}\right)\right\|_{\mathrm{x}}$ where $\left\|\Pi_{\lambda_{j}}\left(\mathrm{P}_{h}\right)\right\| \mathrm{X}$ denotes the norm of $\Pi_{\lambda_{j}}\left(\mathrm{P}_{h}\right)$ when $\operatorname{Ran}\left(\pi_{\eta h^{2}}\left(\mathrm{P}_{h}\right)\right)$ is equipped with $\langle\cdot, \cdot\rangle_{\mathrm{X}}$. Because $\mathrm{P}_{h}$ is self-adjoint on $\operatorname{Ran}\left(\pi_{\eta h^{2}}\left(\mathrm{P}_{h}\right)\right)$ for the Hermitian inner product $\langle\cdot, \cdot\rangle_{\mathbf{X}}$ (see Lemma 14), $\left\|\Pi_{\lambda_{j}}\left(\mathrm{P}_{h}\right)\right\|_{\mathrm{X}}=1$. This concludes the proof of Theorem 2 .

\subsection{Other scalings for the refreshment operator}

In this section, we investigate the effect of a different scaling for the refreshment operator. More precisely, let $\beta \in \mathbb{R}$ be fixed and consider the operators

$$
\mathrm{P}_{h}^{\mathrm{BPS}}(\beta)=-v \cdot \mathrm{d}_{U, h}+\lambda_{1, \mathrm{~J}}(\mathrm{I}-\mathrm{B})+h^{\beta} \lambda_{r}\left(\mathrm{I}-\pi_{v}\right),
$$

and

$$
\mathrm{P}_{h}^{Z Z}(\beta)=-v \cdot \mathrm{d}_{U, h}+\sum_{k=1}^{d} \lambda_{1, J}^{(k)}\left(\mathrm{I}-\mathrm{B}^{(k)}\right)+h^{\beta} \lambda_{r}\left(\mathrm{I}-\pi_{v}\right)
$$


In the following, let

$$
\mathrm{P}_{h}(\beta) \in\left\{\mathrm{P}_{h}^{\mathrm{BPS}}(\beta), \mathrm{P}_{h}^{\mathrm{ZZ}}(\beta)\right\} .
$$

With this notation, the operator $\mathrm{P}_{h}^{\mathrm{BPS}}(0)$ is the operator $\mathrm{P}_{h}$ which has been studied in the previous sections. The antisymmetric part of $\mathrm{P}_{h}(\beta)$ is still equal to $\mathrm{T}_{h}$ (i.e. the antisymmetric part of $\mathrm{P}_{h}$, see the beginning of Section 4.1) whereas its symmetric part now depends on $h$ and equals

$$
\mathrm{S}_{h}(\beta)=\frac{1}{2}\left(\mathrm{P}_{h}(\beta)+\mathrm{P}_{h}^{*}(\beta)\right)=\frac{1}{2}\left(\lambda_{1, \mathrm{~J}}+\lambda_{1, \mathrm{~J}}(\cdot,-\cdot)\right)(\mathrm{I}-\mathrm{B})+h^{\beta} \lambda_{r}\left(\mathrm{I}-\pi_{v}\right) .
$$

Let us recall that Theorems 1 and 2 deal with the case $\beta=0$. When $\beta \neq 0$, the statements of these theorems are changed as follows.

The case when $\beta \geq 0$.

Roughly speaking, when $\beta>0$ (resp. $\beta=0$ ), the refreshment operator $h^{\beta} \lambda_{r}\left(\mathbf{I}-\pi_{v}\right)$ is smaller (resp. of the same order) than the operators $\lambda_{1, \mathrm{~J}}(\mathrm{I}-\mathrm{B})$ or $\sum_{k=1}^{d} \lambda_{1, \mathrm{~J}}^{(k)}\left(\mathrm{I}-\mathrm{B}^{(k)}\right)$. Let $\beta \geq 0$. When $\beta \geq 0$, for $h \in(0,1]$, the symmetric part $\mathrm{S}_{h}(\beta)$ of $\mathrm{P}_{h}(\beta)$ still satisfies $\left\|\mathrm{S}_{h}\right\| \leq c$ for some $c>0$ independent of $h$. Hence, in view of the first three steps of the proof of Proposition 12 (where only $r_{0}$ in (24) is changed into $r_{0} h^{\beta}$ ), there exists $\lambda_{v}>0, C>0$, and $\lambda_{0}>0$, such that for all $u \in \mathcal{C}^{\infty}(\mathrm{E}) \cap \mathrm{G}^{\perp} L^{2}(\mathrm{E})$ and $h \in(0,1]$,

$$
\begin{aligned}
C^{-1} \operatorname{Re}\left\langle\mathrm{P}_{h}(\beta) u,\left[1+\varepsilon\left(\mathrm{A}_{h}+\mathrm{A}_{h}^{*}\right)\right] u\right\rangle_{L^{2}(\mathrm{E})} \geq & \left(\lambda_{v} h^{\beta}-\varepsilon\right)\left\|\left(\mathrm{I}-\pi_{v}\right) u\right\|_{L^{2}(\mathrm{E})}^{2}+\varepsilon \lambda_{x}(h)\left\|\pi_{v} u\right\|_{L^{2}(\mathrm{E})}^{2} \\
& -\varepsilon\left\|\left(\mathrm{I}-\pi_{v}\right) u\right\|_{L^{2}(\mathrm{E})}\left\|\pi_{v} u\right\|_{L^{2}(\mathrm{E})} \\
= & X_{h}(u)^{t} M_{h}(\beta) X_{h}(u),
\end{aligned}
$$

where

$$
X_{h}(u)=\left(\left\|\pi_{v} u\right\|_{L^{2}(\mathrm{E})},\left\|\left(\mathrm{I}-\pi_{v}\right) u\right\|_{L^{2}(\mathrm{E})}\right)^{t}, M_{h}(\beta)=\left(\begin{array}{cc}
\varepsilon \lambda_{x}(h) & -\frac{\varepsilon}{2} \\
-\frac{\varepsilon}{2} & \lambda_{v} h^{\beta}-\varepsilon
\end{array}\right) \text {, with } \lambda_{x}(h)=\lambda_{0} h .
$$

Notice that $M_{h}(0)$ is equal to the matrix $M_{h}$ defined in the fourth step of the proof of Proposition 12. Let us recall that according to [1, Section 3.1 and Lemma 23], the smallest eigenvalue $\Lambda_{0}^{(\beta)}(\varepsilon)$ of $M_{h}(\beta)$ is non negative if $\varepsilon \leq 4 \lambda_{v} h^{\beta} \lambda_{x}(h) /\left(4 \lambda_{x}(h)+1\right)$. From (26) and the lines just before, where one just has to change $\lambda_{v}$ there by $\lambda_{v} h^{\beta}$, one deduces that in the limit $h \rightarrow 0$ :

$$
\varepsilon_{\max }=\lambda_{v} h^{\beta} \times\left[\lambda_{0} h+O\left(h^{2}\right)\right]=\varepsilon_{0} h^{\beta+1}+O\left(h^{\beta+2}\right), \text { where we recall } \varepsilon_{0}=\lambda_{v} \lambda_{0} .
$$

An asymptotic expansion when $h \rightarrow 0$ then leads to

$$
2 \Lambda_{0}^{(\beta)}\left(\varepsilon_{0} h^{\beta+1}\right)=2 \varepsilon_{0} \lambda_{0}\left(1-\frac{\varepsilon_{0}}{4 \lambda_{v} \lambda_{0}}\right) h^{\beta+2}+o\left(h^{\beta+2}\right)=\frac{3}{2} \lambda_{v} \lambda_{0}^{2} h^{\beta+2}+o\left(h^{\beta+2}\right) .
$$

Therefore, when $\beta>0$, Proposition 12 remains valid for $\mathrm{P}_{h}(\beta)$ by changing in its statement all the $h^{2}$ by $h^{\beta+2}$. The same holds for Proposition 13 . In conclusion, when $\beta>0$, Theorem 1 remains valid for $\mathrm{P}_{h}(\beta)$ by changing in its statement $\alpha h^{2}$ by $\alpha h^{\beta+2}$. Finally, Theorem 2 then holds true for $\mathrm{P}_{h}(\beta)$ if one changes there $e^{-\gamma t h^{2}}$ by $e^{-\gamma t h^{\beta+2}}$.

The case when $\beta<0$.

Roughly speaking, in this case, the refreshment operator $h^{\beta} \lambda_{r}\left(\mathbf{I}-\pi_{v}\right)$ is larger than the operators $\lambda_{1, \mathrm{~J}}(\mathrm{I}-\mathrm{B})$ or $\sum_{k=1}^{d} \lambda_{1, \mathrm{~J}}^{(k)}\left(\mathrm{I}-\mathrm{B}^{(k)}\right)$. When $\beta<0$, the symmetric part $\mathrm{S}_{h}(\beta)$ of $\mathrm{P}_{h}(\beta)$ is no more uniformly bounded when in $h \rightarrow 0$ but satisfies, for $h$ small enough, $\left\|S_{h}\right\| \leq c^{\beta}$ for some $c>0$ independent of $h$. Therefore, in view of the first three steps of the proof of Proposition 12 (where 
only $r_{0}$ in (24) is changed into $r_{0} h^{\beta}$ and $\left\|S_{h}\right\|$ is changed into $C h^{\beta}$ in (25)), there exists $\lambda_{v}>0$, $C>0$, and $\lambda_{0}>0$, such that for all $u \in \mathcal{C}^{\infty}(\mathrm{E}) \cap \mathrm{G}^{\perp^{2}(\mathrm{E})}$ and $h$ small enough, it holds:

$$
\begin{aligned}
C^{-1} \operatorname{Re}\left\langle\mathrm{P}_{h}(\beta) u,\left[1+\varepsilon\left(\mathrm{A}_{h}+\mathrm{A}_{h}^{*}\right)\right] u\right\rangle_{L^{2}(\mathrm{E})} \geq & \left(\lambda_{v} h^{\beta}-\varepsilon\right)\left\|\left(\mathrm{I}-\pi_{v}\right) u\right\|_{L^{2}(\mathrm{E})}^{2}+\varepsilon \lambda_{x}(h)\left\|\pi_{v} u\right\|_{L^{2}(\mathrm{E})}^{2} \\
& -\varepsilon h^{\beta}\left\|\left(\mathrm{I}-\pi_{v}\right) u\right\|_{L^{2}(\mathrm{E})}\left\|\pi_{v} u\right\|_{L^{2}(\mathrm{E})} \\
= & X_{h}(u)^{t} M_{h}(\beta) X_{h}(u),
\end{aligned}
$$

where

$$
X_{h}(u)=\left(\left\|\pi_{v} u\right\|_{L^{2}(\mathrm{E})},\left\|\left(\mathrm{I}-\pi_{v}\right) u\right\|_{L^{2}(\mathrm{E})}\right)^{t}, M_{h}(\beta)=\left(\begin{array}{cc}
\varepsilon \lambda_{x}(h) & -\frac{\varepsilon}{2} h^{\beta} \\
-\frac{\varepsilon}{2} h^{\beta} & \lambda_{v} h^{\beta}-\varepsilon
\end{array}\right) \text {, and } \lambda_{x}(h)=\lambda_{0} h .
$$

According to [1, Section 3.1 and Lemma 23] (with $R_{0}=h^{\beta}$ there), for all $h$ small enough, the smallest eigenvalue $\Lambda_{0}(\varepsilon)$ of $M_{h}(\beta)$ is non negative providing that $\varepsilon \leq 4 \lambda_{v} h^{\beta} \lambda_{x}(h) /\left(4 \lambda_{x}(h)+R_{0}^{2}\right)$ and equals

$$
2 \Lambda_{0}^{(\beta)}(\varepsilon)=\lambda_{v} h^{\beta}-\varepsilon\left(1-\lambda_{x}(h)\right)-\sqrt{\left[\lambda_{v} h^{\beta}-\varepsilon\left(1-\lambda_{x}(h)\right)\right]^{2}-4 \varepsilon \lambda_{x}(h)\left(\lambda_{v} h^{\beta}-\varepsilon\right)+\varepsilon^{2} R_{0}^{2}} .
$$

When $h>0$ fixed, from [1, item (b) Lemma 24], the function $\varepsilon \in\left[0,4 \lambda_{x}(h) \lambda_{v} h^{\beta} /\left(4 \lambda_{x}(h)+\right.\right.$ $\left.\left.R_{0}^{2}\right)\right] \mapsto \Lambda_{0}^{(\beta)}(\varepsilon)$ (see 1 , item (b) Lemma 24]) attains its maximum at a unique point $\varepsilon_{\max }$ where

$$
\varepsilon_{\max }=\lambda_{v} h^{\beta} \times \frac{1+\lambda_{x}(h)-\left(1-\lambda_{x}(h)\right)\left[R_{0}^{2} /\left(R_{0}^{2}+4 \lambda_{x}(h)\right)\right]^{\frac{1}{2}}}{\left(1+\lambda_{x}(h)\right)^{2}+R_{0}^{2}}=2 \lambda_{0} \lambda_{v} h^{-3 \beta+1}+o\left(h^{-3 \beta+1}\right),
$$

when $h \rightarrow 0$. Set $\varepsilon_{1}=2 \lambda_{0} \lambda_{v}$. Then, in the limit $h \rightarrow 0$, one has:

$$
2 \Lambda_{0}^{(\beta)}\left(\varepsilon_{1} h^{-3 \beta+1}\right)=2 \lambda_{0} \varepsilon_{1} h^{-3 \beta+2}+o\left(h^{-3 \beta+2}\right)=4 \lambda_{0}^{2} \lambda_{v} h^{-3 \beta+2}+o\left(h^{-3 \beta+2}\right) .
$$

Therefore, when $\beta<0$, Theorem 1 (resp. Theorem 2 remains valid for $\mathrm{P}_{h}(\beta)$ by changing in its statement $\alpha h^{2}$ by $\alpha h^{-3 \beta+2}$ (resp. by changing $e^{-\gamma t h^{2}}$ by $e^{-\gamma t h^{-3 \beta+2}}$ ).

In conclusion, when adding too much or not enough refreshment (i.e $|\beta|>0$ ), our analysis provides a separation when $h \rightarrow 0$ of the spectrum between the $\mathrm{m}_{0}$ smallest eigenvalues of $\mathrm{P}_{h}(\beta)$ and the rest of its spectrum of order $h^{\beta+2}$ if $\beta \geq 0$ or $h^{-3 \beta+2}$ if $\beta<0$. The better separation is thus obtained when $\beta=0$.

\section{Appendix A}

In this appendix, we prove the following non semiclassical result (i.e. when $h>0$ is fixed).

Proposition 16. Let $h>0$ be fixed. The eigenvalue 0 is isolated and has algebraic multiplicity 1 for both $\mathrm{P}_{h}$ and $\mathrm{P}_{h}^{*}$. Moreover, the spectral projection $\pi_{0}\left(\mathrm{P}_{h}\right)$ associated with $\mathrm{P}_{h}$ and 0 equals

$$
u \in L^{2}(\mathrm{E}) \mapsto \pi_{0}\left(\mathrm{P}_{h}\right) u=\frac{\left\langle e^{-\frac{1}{h} U} \mathbf{1}_{\bigvee}, u\right\rangle_{L^{2}(\mathrm{E})}}{\left\|e^{-\frac{1}{h} U} \mathbf{1}_{\mathrm{V}}\right\|_{L^{2}(\mathrm{E})}^{2}} e^{-\frac{1}{h} U} \mathbf{1}_{\bigvee}
$$

and is thus orthogonal in $L^{2}(\mathrm{E})$.

Proof. Since $h>0$ is fixed in what follows, we set $h=1$. Then the computations to prove (22) (which are basically those of [1,14]) imply that for all $\varepsilon_{0}>0$ small enough, there exists $c\left(\varepsilon_{0}\right)>0$ such that for all $u \in \mathcal{C}^{\infty}(\mathrm{E}) \cap\left\{e^{-U} \mathbf{1}_{\mathrm{V}}\right\}^{\perp_{L^{2}(\mathrm{E})}}$,

$$
\operatorname{Re}\left\langle\mathrm{P}_{1} u,\left[1+\varepsilon_{0}\left(\mathrm{~A}_{1}+\mathrm{A}_{1}^{*}\right)\right] u\right\rangle_{L^{2}(\mathrm{E})} \geq c\left(\varepsilon_{0}\right)\|u\|_{L^{2}(\mathrm{E})}^{2} .
$$


Choosing $\varepsilon_{0}>0$ such that $1+\varepsilon_{0}\left(\left\|A_{1}\right\|+\left\|A_{1}^{*}\right\|\right)<2$, Equation 45) implies that for all $u \in$ $\mathcal{C}^{\infty}(\mathrm{E}) \cap\left\{e^{-U} \mathbf{1}_{\mathrm{V}}\right\}^{\perp_{L^{2}(\mathrm{E})}}$ and $z \in \mathbb{C}$, it holds:

$$
\left\|\left(\mathrm{P}_{1}-z\right) u\right\|_{L^{2}(\mathrm{E})} \geq\left[\frac{1}{2} c\left(\varepsilon_{0}\right)-(\operatorname{Re} z)_{+}\right]\|u\|_{L^{2}(\mathrm{E})} .
$$

Set $\mathbf{Z}=\left\|e^{-U} \mathbf{1}_{\mathbf{V}}\right\|_{L^{2}(\mathrm{E})}^{2}$. Then, since $\mathbf{P}_{1}\left(e^{-U} \mathbf{1}_{\mathbf{V}}\right)=\mathrm{P}_{1}^{*}\left(e^{-U} \mathbf{1}_{\mathbf{V}}\right)=0$, for all $u \in \mathcal{C}^{\infty}(\mathbf{E})$, one has:

$$
\left\|\left(\mathrm{P}_{1}-z\right)\left(u-\frac{1}{\mathbf{Z}}\left\langle e^{-U} \mathbf{1}_{\bigvee}, u\right\rangle_{L^{2}(\mathrm{E})} e^{-U} \mathbf{1}_{\mathrm{V}}\right)\right\|_{L^{2}(\mathrm{E})}^{2}=\left\|\left(\mathrm{P}_{1}-z\right) u\right\|_{L^{2}(\mathrm{E})}^{2}-\frac{|z|^{2}}{\mathbf{Z}}\left|\left\langle e^{-U} \mathbf{1}_{\mathrm{V}}, u\right\rangle_{L^{2}(\mathrm{E})}\right|^{2} .
$$

Using (46), it then holds for all $u \in \mathcal{C}^{\infty}(\mathrm{E})$ and $z \in \mathbb{C}$ :

$$
\begin{aligned}
\left\|\left(\mathrm{P}_{1}-z\right) u\right\|_{L^{2}(\mathrm{E})}^{2} \geq & {\left[\frac{1}{2} c\left(\varepsilon_{0}\right)-(\operatorname{Re} z)_{+}\right]^{2}\left\|u-\frac{1}{\mathrm{Z}}\left\langle e^{-U} \mathbf{1}_{\bigvee}, u\right\rangle_{L^{2}(\mathrm{E})} e^{-U} \mathbf{1}_{\bigvee}\right\|_{L^{2}(\mathrm{E})}^{2} } \\
& +\frac{|z|^{2}}{\mathbf{Z}}\left|\left\langle e^{-U} \mathbf{1}_{\mathbf{V}}, u\right\rangle_{L^{2}(\mathrm{E})}\right|^{2}
\end{aligned}
$$

which extends to all $u \in D\left(\mathrm{P}_{1}\right)$. The same estimate holds for $\mathrm{P}_{1}^{*}$ choosing if necessary $\varepsilon_{0}>0$ smaller. Let $z \in \mathbb{C}$ such that $\operatorname{Re}(z)<c\left(\varepsilon_{0}\right) / 2$ and $z \neq 0$. Then, $\mathrm{P}_{1}-z$ is injective with closed and dense range, i.e. $\mathrm{P}_{1}-z$ is invertible. It is in particular is a Fredholm operator (with index $0)$. We claim that $P_{1}$ is also a Fredholm operator. Let us prove it. We have

$$
\text { Ker } \mathrm{P}_{1}=\operatorname{Ker} \mathrm{P}_{1}^{*}=\operatorname{Span}\left(e^{-U} \mathbf{1}_{\mathrm{V}}\right) \text {. }
$$

It is clear that $\operatorname{Span}\left(e^{-U} \mathbf{1}_{\mathbf{V}}\right) \subset \operatorname{Ker} \mathbf{P}_{1} \cap \operatorname{Ker} \mathbf{P}_{1}^{*}$. If now $\mathbf{P}_{1} w=0$, then from Lemma 5 and (2), $\pi_{v} w=w$ is thus independent of $v \in \mathrm{V}$. Then, $\mathrm{P}_{1} w=0$ writes $v \cdot \mathrm{d}_{U, h} w=0$ for all $(x, v) \in \mathrm{M} \times \mathrm{V}$. If $\mathrm{d}_{U, h} w(x) \neq 0$ then choose $v=\mathrm{d}_{U, h} w(x) /\left|\mathrm{d}_{U, h} w(x)\right|$ which leads to $\mathrm{d}_{U, h} w=0$ on $\mathrm{M}$. The same holds for $\mathrm{P}_{1}^{*}$. This proves (48). Furthermore, Equation (47) with $z=0$ implies that the range of $\mathrm{P}_{1}$ is closed. Let us recall that $\left(\operatorname{Ran} \mathrm{P}_{1}\right)^{\perp_{L^{2}(\mathrm{E})}}=\operatorname{Ker} \mathrm{P}_{1}^{*}=\operatorname{Span}\left(e^{-U_{\mathbf{V}}}\right)$. Thus, $\operatorname{Ran} \mathrm{P}_{1}=\operatorname{Span}\left(e^{-U} \mathbf{1}_{\mathrm{V}}\right)^{\perp_{L^{2}(\mathrm{E})}}$, which leads to

$$
\operatorname{dim} \operatorname{coKer} P_{1}=1 \text {. }
$$

Hence, $\mathrm{P}_{1}$ is a Fredholm operator. In conclusion, for all $z \in \mathbb{C}$ such that $\operatorname{Re}(z)<c\left(\varepsilon_{0}\right) / 2, \mathrm{P}_{1}-z$ is a Fredholm operator. Since it is invertible for $z \in \mathbb{R}_{-}^{*}\left(\mathrm{P}_{1}\right.$ is $m$-accretive), by the analytic Fredholm Theorem, the function

$$
z \in\left\{\tilde{z} \in \mathbb{C}, \operatorname{Re}(\tilde{z})<c\left(\varepsilon_{0}\right) / 2\right\} \mapsto\left(\mathrm{P}_{1}-z\right)^{-1}
$$

is meromorphic with poles of finite rank. The only pole of this function in this region is $z=0$ and has therefore finite algebraic multiplicity. Let us assume that for $\mathrm{P}_{1}$ this algebraic multiplicity is strictly larger than 1 . Since $\operatorname{Ker}\left(\mathbf{P}_{1}\right)=\operatorname{Span}\left(e^{-U} \mathbf{1}_{\mathbf{V}}\right)$, this implies that there exists a generalized eigenfunction $f \in D\left(\mathrm{P}_{1}\right)$ such that $\mathrm{P}_{1} f=e^{-U} \mathbf{1}_{\mathrm{V}}$. Consequently $0<\left\|e^{-U} \mathbf{1}_{\mathbf{V}}\right\|_{L^{2}(\mathrm{E})}^{2}=\left\langle\mathrm{P}_{1} f, e^{-U} \mathbf{1}_{\mathbf{V}}\right\rangle=\left\langle f, \mathrm{P}_{1}^{*}\left(e^{-U} \mathbf{1}_{\mathbf{V}}\right)\right\rangle=0$ which leads to a contradiction. The same reasoning also applies to $\mathrm{P}_{1}^{*}$. This concludes the proof of Proposition 16 .

Let us end this work with a short remark on how one can easily deduce the convergence of $\left(e^{-t \mathrm{P}_{1}}\right)_{t \geq 0}$ to $\pi_{0}\left(\mathrm{P}_{1}\right)$ as $t \rightarrow \infty$ from (46) and with the Gearhart-Prüss Theorem. Using (46), for all $u \in \mathcal{C}^{\infty}(\mathrm{E}) \cap\left\{e^{-U_{\mathrm{V}}}\right\}^{\perp_{L^{2}(\mathrm{E})}}$ and $z \in \mathbb{C}$ such that Re $z \leq c\left(\varepsilon_{0}\right) / 4$, one has:

$$
\left\|\left(\mathrm{P}_{1}-z\right) u\right\|_{L^{2}(\mathrm{E})} \geq \frac{1}{4} c\left(\varepsilon_{0}\right)\|u\|_{L^{2}(\mathrm{E})} .
$$


This implies that the resolvent of $\left.\mathrm{P}_{1}\right|_{\left(1-\pi_{0}\left(\mathrm{P}_{1}\right)\right) L^{2}(\mathrm{E})}$ is uniformly bounded on the set $\{z, \operatorname{Re} z<$ $\left.c\left(\varepsilon_{0}\right) / 4\right\}$. Hence, applying the Gearhart-Prüss Theorem to $e^{-t \mathrm{P}_{1}}\left(1-\pi_{0}\left(\mathrm{P}_{1}\right)\right)$ on $\operatorname{Ran}\left(1-\pi_{0}\left(\mathrm{P}_{1}\right)\right)$, it holds for $t \geq 0$,

$$
\left\|e^{-t \mathrm{P}_{1}}\left(1-\pi_{0}\left(\mathrm{P}_{1}\right)\right)\right\| \leq C e^{-\frac{c\left(\varepsilon_{0}\right)}{4} t},
$$

for some $C>0$ independent of $t \geq 0$. In conclusion, this shows that starting from (45), we recover the (non semiclassical) results of 1,14 with the Gearhart-Prüss Theorem together with uniform resolvent estimates.

\section{Appendix B}

In this appendix, we give an upper bound on $\lambda_{2}$. To this end consider the case when $U$ has two local minima $x_{1}$ and $x_{2}$ (namely $U$ is a double-well potential: $\mathrm{m}_{0}=2$ ) with $U\left(x_{1}\right)<U\left(x_{2}\right)$. Define, for $j=1,2$ (see Section 3 ):

$$
\psi_{j, h}=\frac{\pi_{\eta h^{2}}\left(\mathrm{P}_{h}\right)\left(\Phi_{j, h} \mathbf{1}_{\mathbf{V}}\right)}{\mathrm{Z}_{j}} \text { with } \mathrm{Z}_{j}=\left\|\pi_{\eta h^{2}}\left(\mathrm{P}_{h}\right)\left(\Phi_{j, h} \mathbf{1}_{\mathbf{V}}\right)\right\|_{\mathbf{x}}
$$

It holds,

$$
\left\|\mathrm{d}_{U, h}\left(\Phi_{2, h}\right)\right\|_{L^{2}(\mathrm{M})}^{2}=\mu_{2}
$$

where $\mu_{2}>0$ is the second smallest eigenvalue of the Witten Laplacian $\Delta_{U, h}$ on M. Since $\mathrm{P}_{h}\left(\Phi_{2, h} 1_{\mathrm{V}}\right)=-v \cdot \mathrm{d}_{U, h}\left(\Phi_{2, h}\right) 1_{\mathrm{V}}$, for $h$ small enough, one has:

$$
\left\|\mathrm{P}_{h}\left(\Phi_{2, h} 1_{\mathrm{V}}\right)\right\|_{L^{2}(\mathrm{E})}^{2} \leq \mu_{2} .
$$

Moreover, $\pi_{\eta h^{2}}\left(\mathrm{P}_{h}\right)\left(\Phi_{1, h} \mathbf{1}_{\vee}\right)=\Phi_{1, h} \mathbf{1}_{\bigvee}$ because $\mathrm{P}_{h}\left(\Phi_{1, h} \mathbf{1}_{\vee}\right)=0$ (recall that $\Phi_{1, h}=$ $\left.e^{-\frac{1}{h} U} /\left\|e^{-\frac{1}{h} U}\right\|_{L^{2}(\mathrm{M})}\right)$. From 42, $\left(\psi_{1, h}, \psi_{2, h}\right)$ is a quasi-orthonormal basis of $\operatorname{Ran} \pi_{\eta h^{2}}\left(\mathrm{P}_{h}\right)$ (equipped with the Hermitian inner product $\langle\cdot, \cdot\rangle_{\mathbf{X}}$ ). Consequently, $\lambda_{2}$ is the largest eigenvalue of the symmetric matrix $\mathbf{N}_{h}=\left(\left\langle\mathrm{P}_{h} \psi_{i, h}, \psi_{j, h}\right\rangle_{\mathbf{X}}\right)_{i, j=1,2}$. One has, $\mathbf{N}_{h}=\left(0,0 ; 0,\left\langle\mathrm{P}_{h} \psi_{2, h}, \psi_{2, h}\right\rangle_{\mathbf{X}}\right)$ and therefore, $\lambda_{2}=\left\langle\mathrm{P}_{h} \psi_{2, h}, \psi_{2, h}\right\rangle_{\mathbf{X}}$. It holds, since $\pi_{\eta h^{2}}\left(\mathrm{P}_{h}\right)$ is symmetric for the Hermitian inner product $\langle\cdot, \cdot\rangle_{\mathbf{X}}$,

$$
\begin{aligned}
\mathrm{Z}_{2}^{2}\left\langle\mathrm{P}_{h} \psi_{2, h}, \psi_{2, h}\right\rangle \mathrm{x} & =\left\langle\mathrm{P}_{h}\left(\Phi_{2, h} \mathbf{1}_{\mathrm{V}}\right), \Phi_{2, h} \mathbf{1}_{\mathrm{V}}\right\rangle_{\mathbf{x}}+O\left(\left\|\mathrm{P}_{h}\left(\Phi_{2, h} \mathbf{1}_{\mathrm{V}}\right)\right\|_{L^{2}(\mathrm{E})}\left\|\left(1-\pi_{\eta h^{2}}\left(\mathrm{P}_{h}\right)\right)\left(\Phi_{2, h} \mathbf{1}_{\mathrm{V}}\right)\right\|_{L^{2}(\mathrm{E})}\right) \\
& =0+O\left(h^{-2}\left\|\mathrm{P}_{h}\left(\Phi_{2, h} \mathbf{1}_{\mathrm{V}}\right)\right\|_{L^{2}(\mathrm{E})}^{2}\right)
\end{aligned}
$$

where we used the equality $1-\pi_{\eta h^{2}}\left(\mathrm{P}_{h}\right)=\frac{-1}{2 \pi \mathrm{i}} \int_{|z|=\eta h^{2}} z^{-1}\left(z-\mathrm{P}_{h}\right)^{-1} \mathrm{P}_{h} d z$ together with item 1 in Proposition 13 . Notice that $\left\langle\mathrm{P}_{h}\left(\Phi_{2, h} \mathbf{1}_{\mathbf{V}}\right), \Phi_{2, h} \mathbf{1}_{\mathbf{V}}\right\rangle_{\mathbf{X}}=0$ because $\int_{\mathbb{V}} v_{i} d \nu=0$ for all $i \in\{1, \ldots, d\}$. Thus, using (49) and 42), one deduces that for $h$ small enough:

$$
\lambda_{2} \leq C h^{-2} \mu_{2},
$$

for some $C>0$ independent of $h$. We refer to $10,20,28,33,34$ for asymptotic equivalents of $\mu_{2}$ or for sharp lower and upper bounds on $\mu_{2}$. In particular, from 10,20,33, it holds in the limit $h \rightarrow 0: \mu_{2}=A h e^{-\frac{2}{h}\left(U\left(z_{*}\right)-U\left(x_{2}\right)\right)}(1+O(h))$ for some $A>0$ and where $z_{*}$ is a saddle point of $U$ connecting $x_{1}$ and $x_{2}$. Thus, for $h$ small enough,

$$
\lambda_{2} \leq C h^{-1} e^{-\frac{2}{h}\left(U\left(z_{*}\right)-U\left(x_{2}\right)\right)} .
$$

Thus, $\lim _{h \rightarrow 0} h \ln \lambda_{2} \leq-2\left(U\left(z_{*}\right)-U\left(x_{2}\right)\right)$. We expect $\lambda_{2}$ to satisfy $\lim _{h \rightarrow 0} h \ln \lambda_{2}=-2\left(U\left(z_{*}\right)-\right.$ $\left.U\left(x_{2}\right)\right)$ as suggested in 36, Theorem 1.1]. To this end, one needs to construct a quasi-mode $\tilde{\psi}_{2, h}$ so that $\left\langle\mathrm{P}_{h} \tilde{\psi}_{2, h}, \tilde{\psi}_{2, h}\right\rangle \mathbf{x} \neq 0,\left\langle\mathrm{P}_{h} \tilde{\psi}_{2, h}, \tilde{\psi}_{2, h}\right\rangle \mathbf{x}$ gives the expected asymptotic equivalent of $\lambda_{2}$, and $\left\|\mathrm{P}_{h}\left(\Phi_{2, h} \mathbf{1}_{\mathrm{V}}\right)\right\|_{L^{2}(\mathrm{E})}=o\left(h^{2}\right)\left|\left\langle\mathrm{P}_{h} \tilde{\psi}_{2, h}, \tilde{\psi}_{2, h}\right\rangle \mathrm{x}\right|$. This will be the purpose of a future work. 
Acknowledgement. The second author is grateful to Dorian Le Peutrec for his suggestion in the proof of Proposition 6. This work is supported by the ANR-17-CE40-0030 - EFI - Entropy, flows, inequalities.

\section{References}

[1] C. Andrieu, A. Durmus, N. Nüsken, and J. Roussel. Hypercoercivity of Piecewise Deterministic Markov Process-Monte Carlo. ArXiv version : 1808.08592, 2018.

[2] R. Azaïs, J. B. Bardet, A. Génadot, N. Krell, and P-A. Zitt. Piecewise Deterministic Markov Process - recent results. In ESAIM: Proceedings, volume 44, pages 276-290. EDP Sciences, 2014.

[3] J. Bierkens. Non-reversible Metropolis-Hastings. Statistics and Computing, 26(6):1213-1228, 2016.

[4] J. Bierkens, A. Bouchard-Côté, A. Doucet, A. B. Duncan, P. Fearnhead, T. Lienart, G. Roberts, and S. J. Vollmer. Piecewise deterministic Markov processes for scalable Monte Carlo on restricted domains. Statistics \& Probability Letters, 136:148-154, 2018.

[5] J. Bierkens, P. Fearnhead, and G. Roberts. The zig-zag process and super-efficient sampling for Bayesian analysis of big data. The Annals of Statistics, 47(3):1288-1320, 2019.

[6] J. Bierkens, K. Kamatani, and G. Roberts. High-dimensional scaling limits of piecewise deterministic sampling algorithms. arXiv:180\%.11358, 2018.

[7] J. Bierkens and S. M V. Lunel. Spectral analysis of the zigzag process. ArXiv version : 1905.01691, 2019.

[8] J. Bierkens, G. O Roberts, and P-A. Zitt. Ergodicity of the zigzag process. The Annals of Applied Probability, 29(4):2266-2301, 2019.

[9] A. Bouchard-Côté, S. J. Vollmer, and A. Doucet. The bouncy particle sampler: A nonreversible rejection-free markov chain Monte Carlo method. Journal of the American Statistical Association, 113(522):855-867, 2018.

[10] A. Bovier, V. Gayrard, and M. Klein. Metastability in reversible diffusion processes. II. Precise asymptotics for small eigenvalues. Journal of the European Mathematical Society, 7(1):69-99, 2005.

[11] H. Brezis. Functional analysis, Sobolev spaces and partial differential equations. Springer Science \& Business Media, 2010.

[12] M. H. A Davis. Piecewise-deterministic Markov processes: A general class of non-diffusion stochastic models. Journal of the Royal Statistical Society: Series B (Methodological), 46(3):353-376, 1984.

[13] G. Deligiannidis, A. Bouchard-Côté, and A. Doucet. Exponential Ergodicity of the Bouncy Particle Sampler. The Annals of Statistics, 47(3):1268-1287, 2019.

[14] J. Dolbeault, C. Mouhot, and C. Schmeiser. Hypocoercivity for linear kinetic equations conserving mass. Transactions of the American Mathematical Society, 367(6):3807-3828, 2015.

[15] A. Durmus, A. Guillin, and P. Monmarché. Piecewise Deterministic Markov Processes and their invariant measure. ArXiv version : 1807.05421, 2018.

[16] A. Durmus, A. Guillin, and P. Monmarché. Geometric ergodicity of the Bouncy Particle Sampler. To appear in Annals of Applied Probability, 2019.

[17] A. Faggionato, D. Gabrielli, and M. Ribezzi-Crivellari. Non-equilibrium thermodynamics of piecewise deterministic Markov processes. Journal of Statistical Physics, 137(2):259, 2009.

[18] P. Fearnhead, J. Bierkens, M. Pollock, and G. O. Roberts. Piecewise deterministic Markov processes for continuous-time Monte Carlo. Statistical Science, 33(3):386-412, 2018.

[19] B. Helffer. Spectral theory and its applications, volume 139. Cambridge University Press, 2013.

[20] B. Helffer, M. Klein, and F. Nier. Quantitative analysis of metastability in reversible diffusion processes via a Witten complex approach. Matemática Contemporânea, 26:41-85, 2004.

[21] B. Helffer and F. Nier. Hypoelliptic Estimates and Spectral Theory for Fokker-Planck Operators and Witten Laplacians, volume 1862 of Lecture Notes in Mathematics. Springer, 2005.

[22] B. Helffer and J. Sjöstrand. Puits multiples en mécanique semi-classique IV Etude du complexe de Witten. Communication in Partial Differential Equations, 10(3):245-340, 1985.

[23] B. Helffer and J. Sjöstrand. From resolvent bounds to semigroup bounds. Arxiv version : 1001.4171, 2010. 
[24] F. Hérau. Hypocoercivity and exponential time decay for the linear inhomogeneous relaxation Boltzmann equation. Asymptotic Analysis, 46(3, 4):349-359, 2006.

[25] F. Hérau, M. Hitrik, and J. Sjöstrand. Tunnel effect and symmetries for Kramers-Fokker-Planck type operators. Journal of the Institute of Mathematics of Jussieu, 10(3):567-634, 2011.

[26] F. Hérau and F. Nier. Isotropic hypoellipticity and trend to equilibrium for the Fokker-Planck equation with a high-degree potential. Archive for Rational Mechanics and Analysis, 171(2):151-218, 2004.

[27] P. Holderrieth. Cores for Piecewise-Deterministic Markov Processes used in Markov Chain Monte Carlo. ArXiv version : 1910.11429v2, 2019.

[28] R.A. Holley, S. Kusuoka, and D.W. Stroock. Asymptotics of the spectral gap with applications to the theory of simulated annealing. Journal of Functional Analysis, 83(2):333-347, 1989.

[29] T. Kato. Perturbation theory for linear operators, volume 132. Springer Science \& Business Media, 2013.

[30] D. Le Peutrec and L. Michel. Sharp spectral asymptotics for non-reversible metastable diffusion processes. Arxiv version: 190\%.09166, 2019.

[31] T. Lelièvre and G. Stoltz. Partial differential equations and stochastic methods in molecular dynamics. Acta Numerica, 25:681-880, 2016.

[32] N. Metropolis, A.W. Rosenbluth, M.N. Rosenbluth, A. Teller, and E. Teller. Equation of state calcultations by fast computing machines. The Journal of Chemical Physics, 21(6):1087, 1953.

[33] L. Michel. About small eigenvalues of the Witten Laplacian. Pure and Applied Analysis, 1(2):149-206, 2019.

[34] L. Miclo. Comportement de spectres d'opérateurs de Schrödinger à basse température. Bulletin des Sciences Mathématiques, 119(6):529-554, 1995.

[35] L. Miclo and P. Monmarché. Étude spectrale minutieuse de processus moins indécis que les autres. In Séminaire de Probabilités XLV, pages 459-481. Springer, 2013.

[36] P. Monmarché. Piecewise deterministic simulated annealing. ALEA, Latin American Journal of Probability and Mathematical Statistics, 13(1):357-398, 2014.

[37] G.K. Pedersen. Analysis Now, volume 118. Springer Science \& Business Media, 2012.

[38] E. A. J. F. Peters and G. de With. Rejection-free Monte Carlo sampling for general potentials. Physical Review E, 85(2):026703, 2012.

[39] V. Robbe. Small eigenvalues of the low temperature linear relaxation Boltzmann equation with a confining potential. In Annales Henri Poincaré, volume 17, pages 937-952. Springer, 2016.

[40] J. Sjöstrand and M. Zworski. Elementary linear algebra for advanced spectral problems. In Annales de l'institut Fourier, volume 57, pages 2095-2141, 2007.

[41] O. Staffans. Well-posed linear systems, volume 103. Cambridge University Press, 2005. 